\title{
Aryl Sulfoxides via Palladium-Catalyzed Arylation of Sulfenate Anions
}

Guillaume Maitro, Sophie Vogel, Guillaume Prestat, David Madec, ${ }^{*}$ and Giovanni Poli*

Université Pierre et Marie Curie-Paris 6, Laboratoire de Chimie organique (UMR CNRS 7611), Institut de Chimie Moléculaire (FR 2769), Case 183, 4 Place Jussieu, F-75252, Paris Cedex 05, France giovanni.poli@upmc.fr; david.madec@upmc.fr

\section{Supporting Information}

\section{Table of contents}

(I) General remarks $\quad 2$

(II) Experimental procedure and characterization data for $\beta$-sulfinyl ester 1c 2

(III) General procedure for palladium-catalyzed arylation of sulfenate anions under biphasic conditions

(IV) Characterization data for aromatic sulfoxides starting from 1a 4

(V) Characterization data for aromatic sulfoxides starting from 1b 10

(VI) Characterization data for aromatic sulfoxides starting from 1c $\quad 11$

(VII) Characterization data for aromatic sulfoxides starting from 1d 12

(VIII) Experimental procedure for the pseudo-domino arylation/Mizoroki-Heck sequence and characterization data for compound $16 \quad 13$

(IX) spectral data 14 


\section{(I) General remarks}

Dichloromethane was distilled from $\mathrm{CaH}_{2}$. All other reagents and solvents were used without further purification. ${ }^{1} \mathrm{H}$ NMR (400 MHz) and ${ }^{13} \mathrm{C}$ NMR (100 MHz) were recorded on a Brüker ARX-400 spectrometer using the residual peak of chloroform- $d$ as internal standard. Chemical shifts are reported in ppm. IR spectra were recorded on a Brüker Tensor 27 (pike) instrument and only the strongest or structurally most important peaks were listed. Chromatographic purifications were conducted using 40-63 $\mu \mathrm{m}$ silica gel and analytical TLC were performed on Merck precoated silica $60-\mathrm{F}_{254}$ plates.

\section{(II) Experimental procedure and characterization data for $\beta$-sulfinyl ester 1c}

According to Maitro, G.; Prestat, G.; Madec, D.; Poli, G. J. Org. Chem. 2006, 71, 7449-7454.

To a solution of thiol $(20 \mathrm{mmol})$ and potassium carbonate $(5 \mathrm{~mol} / \mathrm{o})$ in dichloromethane $(5$ $\mathrm{mL}$ ) was added rapidly tert-butyl acrylate $(3.2 \mathrm{~mL}, 22 \mathrm{mmol})$. After stirring for $20 \mathrm{~h}$ at room temperature, water was added to the mixture and the organic layer was washed with successively water $(2 \times 30 \mathrm{~mL})$ and brine $(2 \times 30 \mathrm{~mL})$, dried over anhydrous $\mathrm{MgSO}_{4}$ and evaporated under reduced pressure. The product was obtained as a colorless oil with $94 \%$ yield and was used without further purification.

\section{3-benzylsulfanyl-propionic acid tert-butyl ester:}

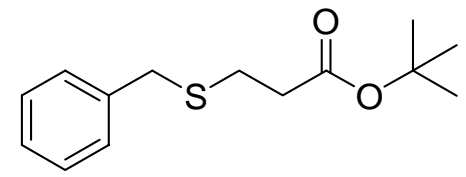

${ }^{1} \mathrm{H} \mathrm{NMR}\left(\mathrm{CDCl}_{3}, 400 \mathrm{MHz}\right): \delta$ 7.35-7.30 (m, 4H), 7.28-7.24 (m, 1H), $3.74(\mathrm{~s}, 2 \mathrm{H}), 2.67(\mathrm{t}, J$ $=7.1 \mathrm{~Hz}, 2 \mathrm{H}), 2.48(\mathrm{t}, J=7.3 \mathrm{~Hz}, 2 \mathrm{H}), 1.47(\mathrm{~s}, 9 \mathrm{H})$.

${ }^{13} \mathrm{C} \mathrm{NMR}\left(\mathrm{CDCl}_{3}, 100 \mathrm{MHz}\right): \delta 171.1,138.2,128.8,128.5,127.0,80.6,36.2,35.6,28.0,26.4$. IR (neat): $v 2977,1725,1366,1250,1147,845,698 \mathrm{~cm}^{-1}$.

$\mathrm{MS}\left(\mathrm{CI} / \mathrm{NH}_{3}\right): m / z 270\left(\mathrm{MNH}_{4}^{+}\right), 253\left(\mathrm{MH}^{+}\right), 214\left[\left(\mathrm{MNH}_{4}{ }^{+}\right)-(t-\mathrm{Bu})\right], 197\left[\left(\mathrm{MH}^{+}\right)-(t-\mathrm{Bu})\right]$.

Anal. Calcd for $\mathrm{C}_{14} \mathrm{H}_{20} \mathrm{O}_{2} \mathrm{~S}$ : C, 66.63; H, 7.99. Found: C, 66.29; H, 8.31. 
A dry dichloromethane solution $(25 \mathrm{~mL})$ of preceding thioether $(10 \mathrm{mmol})$ was added to a suspension of wet neutral alumine oxide (10 g, ratio alumina oxide/water: $5 \mathrm{~g}$ for $1 \mathrm{~mL}$ ) and Oxone $^{\circledR}$ in dry dichloromethane $(25 \mathrm{~mL})$ at room temperature. The reaction mixture was refluxed for $3 \mathrm{~h}$, then cooled to room temperature, filtered and evaporated under reduced pressure. Purification by silica gel flash chromatography (cyclohexane/ethyl acetate, 3:2 $\rightarrow$ $1: 1 \rightarrow 3: 7)$ followed by recrystallization from $n$-hexane to afford the product as a white solid (81\% yield).

\section{3-phenylmethylsulfinyl-propionic acid tert-butyl ester 1c:}

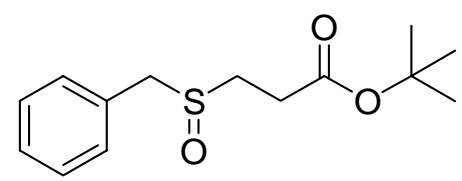

${ }^{1} \mathrm{H}$ NMR $\left(\mathrm{CDCl}_{3}, 400 \mathrm{MHz}\right): \delta$ 7.34-7.24 (m, 5H), $3.94(\mathrm{AB}, J=12.9 \mathrm{~Hz}, 2 \mathrm{H}), 2.91-2.81(\mathrm{~m}$, $1 \mathrm{H}), 2.71-2.63(\mathrm{~m}, 3 \mathrm{H}), 1.38(\mathrm{~s}, 9 \mathrm{H})$.

${ }^{13} \mathrm{C} \mathrm{NMR}\left(\mathrm{CDCl}_{3}, 100 \mathrm{MHz}\right): \delta 170.3,129.9,129.5,128.9,128.3,81.3,58.3,45.6,27.9(2 \mathrm{C})$. IR (powder): $v$ 2963, 1727, 1364, 1235, 1156, 1037, $774 \mathrm{~cm}^{-1}$. MS (CI/NH $): m / z 269\left(\mathrm{MH}^{+}\right), 213\left[\left(\mathrm{MH}^{+}\right)-(t-\mathrm{Bu})\right]$.

Anal. Calcd for $\mathrm{C}_{14} \mathrm{H}_{20} \mathrm{O}_{3} \mathrm{~S}: \mathrm{C}, 62.66 ; \mathrm{H}, 7.51 ; \mathrm{S}, 11.95$. Found: $\mathrm{C}, 62.55 ; \mathrm{H}, 7.79 ; \mathrm{S}, 12.12$. M.p. $64-66{ }^{\circ} \mathrm{C}$.

(III) General procedure for palladium-catalyzed arylation of sulfenate anions under biphasic conditions

To a solution of tris(dibenzylideneacetone)dipalladium $(5 \mathrm{~mol} \%)$ in toluene $(500 \mu \mathrm{L})$ was added xantphos (10 mol\%). The solution was stirred at room temperature for $5 \mathrm{~min}$. Then, a solution of iodide substrate $(0.6 \mathrm{mmol}$ in $1.5 \mathrm{~mL}$ of toluene), $\beta$-sulfinylester $(0.5 \mathrm{mmol}$ in 1.5 $\mathrm{mL}$ of toluene), $3.5 \mathrm{~mL}$ of distilled water and $50 \%$ aqueous $\mathrm{KOH}$ solution $(10 \mathrm{mmol})$ were successively added. The resulting biphasic system was stirred vigorously at $70{ }^{\circ} \mathrm{C}$ for $2 \mathrm{~h}$. Then, after cooling to room temperature, the aqueous phase was extracted twice with dichloromethane, the collected organic layers were dried over anhydrous $\mathrm{MgSO}_{4}$ and the 
solvent was removed under reduced pressure. The crude product was purified by flash chromatography.

(IV) Characterization data for aromatic sulfoxides starting from 1a

\section{p-anisyl-p-tolyl sulfoxide 2a:}<smiles>COc1ccc(S(=O)c2ccc(C)cc2)cc1</smiles>

Purification by silica gel flash chromatography (cyclohexane/ethyl acetate, 9:1 $\rightarrow 85: 15$ ) to afford the product as a pale yellow solid ( $70 \%$ yield).

${ }^{1} \mathrm{H}$ NMR $\left(\mathrm{CDCl}_{3}, 400 \mathrm{MHz}\right): \delta$ 7.58-7.55 (m, 2H), 7.52-7.50 (m, 2H), $7.26(\mathrm{~d}, J=8.1 \mathrm{~Hz}$, 2H), 6.98-6.94 (m, 2H), $3.82(\mathrm{~s}, 3 \mathrm{H}), 2.38(\mathrm{~s}, 3 \mathrm{H})$.

${ }^{13} \mathrm{C} \mathrm{NMR}\left(\mathrm{CDCl}_{3}, 100 \mathrm{MHz}\right): \delta 161.9,142.7,141.3,137.0,130.0,127.1,124.8,114.8,55.5$, 21.4 .

IR (powder): $v$ 2921, 1592, 1493, 1248, 1036, $809 \mathrm{~cm}^{-1}$.

MS (CI/NH3): $m / z 247\left(\mathrm{MH}^{+}\right)$.

HRMS $m / z$ calculated for $\mathrm{C}_{14} \mathrm{H}_{14} \mathrm{O}_{2} \mathrm{~S}\left(\mathrm{M}^{+}\right): 246.0715$. Found: 246.0729 .

M.p. $50-52^{\circ} \mathrm{C}$.

\section{bis(p-tolyl) sulfoxide 3a:}

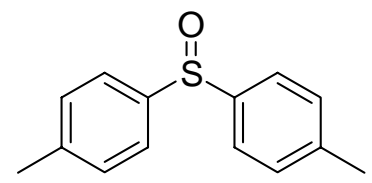

Purification by silica gel chromatography (cyclohexane/ethyl acetate, 9:1 $\rightarrow 85: 15$ ) to give a white solid ( $81 \%$ yield).

Spectral data in agreement with those already reported by Laali, K. K.; Nagvekar, D. S. J. Org. Chem. 1991, 56, 1867-1874 (and commercially available). 
${ }^{1} \mathrm{H}$ NMR $\left(\mathrm{CDCl}_{3}, 400 \mathrm{MHz}\right): \delta 7.53(\mathrm{~d}, J=8.1 \mathrm{~Hz}, 4 \mathrm{H}), 7.26(\mathrm{~d}, J=8.1 \mathrm{~Hz}, 4 \mathrm{H}), 2.38(\mathrm{~s}$, $6 \mathrm{H})$.

${ }^{13} \mathrm{C}$ NMR (CDCl $\left.3,100 \mathrm{MHz}\right): \delta 142.8,141.5,130.1,125.0,21.5$.

IR (neat): $v$ 2920, 1492, 1088, 1038, 1014, $805 \mathrm{~cm}^{-1}$.

$\operatorname{MS}\left(\mathrm{CI} / \mathrm{NH}_{3}\right): m / z 461,248\left(\mathrm{MNH}_{4}{ }^{+}\right), 231\left(\mathrm{MH}^{+}\right)$.

\section{m-anisyl-p-tolyl sulfoxide 4a:}<smiles>COc1cccc(S(=O)c2ccc(C)cc2)c1</smiles>

Purification by silica gel flash chromatography (cyclohexane/ethyl acetate, 9:1 $\rightarrow$ 85:15) to afford a brown oil (63\% yield).

${ }^{1} \mathrm{H}$ NMR $\left(\mathrm{CDCl}_{3}, 400 \mathrm{MHz}\right): \delta$ 7.56-7.54 (m, 2H), $7.35(\mathrm{t}, J=7.8 \mathrm{~Hz}, 1 \mathrm{H}), 7.26-7.24(\mathrm{~m}$, $3 \mathrm{H}), 7.17-7.14(\mathrm{~m}, 1 \mathrm{H}), 6.97-6.94(\mathrm{~m}, 1 \mathrm{H}), 3.84(\mathrm{~s}, 3 \mathrm{H}), 2.38(\mathrm{~s}, 3 \mathrm{H})$.

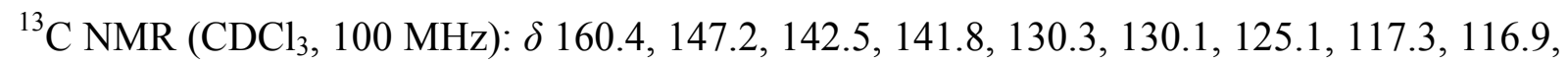
109.0, 55.6, 21.5.

IR (neat): $v 2923,1593,1479,1232,1035,684 \mathrm{~cm}^{-1}$.

HRMS $m / z$ calculated for $\mathrm{C}_{14} \mathrm{H}_{14} \mathrm{O}_{2} \mathrm{~S}\left(\mathrm{M}^{+}\right): 246.0715$. Found: 246.0729 .

\section{o-anisyl-p-tolyl sulfoxide 5a:}<smiles>COc1ccccc1S(=O)c1ccc(C)cc1</smiles>

Purification by silica gel flash chromatography (cyclohexane/ethyl acetate 9:1 $\rightarrow$ 85:15) followed by recrystallization from $n$-hexane to give colourless crystals ( $79 \%$ yield).

${ }^{1} \mathrm{H}$ NMR $\left(\mathrm{CDCl}_{3}, 400 \mathrm{MHz}\right): \delta 7.93(\mathrm{dd}, J=7.8,1.8 \mathrm{~Hz}, 1 \mathrm{H}), 7.60(\mathrm{~d}, J=8.1 \mathrm{~Hz}, 2 \mathrm{H}), 7.41$ (ddd, $J=8.1,7.6,1.8 \mathrm{~Hz}, 1 \mathrm{H}), 7.23$ (d, $J=8.1 \mathrm{~Hz}, 2 \mathrm{H}), 7.17$ (ddd, $J=7.8,7.6,1.0 \mathrm{~Hz}, 1 \mathrm{H}$ ), $6.86(\mathrm{~d}, J=8.1 \mathrm{~Hz}, 1 \mathrm{H}), 3.81(\mathrm{~s}, 3 \mathrm{H}), 2.37(\mathrm{~s}, 3 \mathrm{H})$. 
${ }^{13} \mathrm{C} \mathrm{NMR}\left(\mathrm{CDCl}_{3}, 100 \mathrm{MHz}\right): \delta 155.7,142.4,141.4,133.4,132.1,129.8,125.5,124.7,121.7$, 111.1, 55.8, 21.6.

IR (powder): $v$ 2921, 1477, 1273, 1030, $762 \mathrm{~cm}^{-1}$.

Anal. Calcd for $\mathrm{C}_{14} \mathrm{H}_{14} \mathrm{O}_{2} \mathrm{~S}$ : C, 68.26; H, 5.73; S, 13.02. Found: C, 67.86; H, 5.77; S, 13.32 .

M.p. $88-90{ }^{\circ} \mathrm{C}$.

\section{p-acetophenyl-p-tolyl sulfoxide 6a:}<smiles>CC(=O)c1ccc(S(=O)c2ccc(C)cc2)cc1</smiles>

Purification by silica gel chromatography (cyclohexane/ethyl acetate, 9:1 $\rightarrow$ 85:15) to afford the product as a pale yellow solid (58\% yield).

${ }^{1} \mathrm{H}$ NMR ( $\left.\mathrm{CDCl}_{3}, 400 \mathrm{MHz}\right): \delta 8.01(\mathrm{~d}, J=8.3 \mathrm{~Hz}, 2 \mathrm{H}), 7.72(\mathrm{~d}, J=8.3 \mathrm{~Hz}, 2 \mathrm{H}), 7.54(\mathrm{~d}, J=$ $8.3 \mathrm{~Hz}, 2 \mathrm{H}), 7.27$ (d, J=8.1 Hz, 2H), 2.60 (s, 3H), 2.36 (s, 3H).

${ }^{13} \mathrm{C} \mathrm{NMR}\left(\mathrm{CDCl}_{3}, 100 \mathrm{MHz}\right): \delta 197.2,150.9,142.4,141.9,138.8,130.3,129.1,125.2,124.6$, $26.9,21.5$

IR (powder): $v$ 2922, 1681, 1257, 1049, 806, $615 \mathrm{~cm}^{-1}$.

MS (CI/NH$): m / z 276\left(\mathrm{MNH}_{4}{ }^{+}\right), 259\left(\mathrm{MH}^{+}\right), 243$.

HRMS $m / z$ calculated for $\mathrm{C}_{15} \mathrm{H}_{14} \mathrm{O}_{2} \mathrm{~S}\left(\mathrm{M}^{+}\right)$: 258.0715. Found: 258.0712 .

M.p. $102-104{ }^{\circ} \mathrm{C}$.

p-nitrophenyl-p-tolyl sulfoxide 7a:<smiles>Cc1ccc(S(=O)c2ccc([N+](=O)[O-])cc2)cc1</smiles>

Purification by silica gel flash chromatography (cyclohexane/ethyl acetate, 9:1 $\rightarrow$ 85:15) to afford the product as a pale yellow solid ( $82 \%$ yield). 
${ }^{1} \mathrm{H}$ NMR $\left(\mathrm{CDCl}_{3}, 400 \mathrm{MHz}\right): \delta 8.32(\mathrm{~d}, J=8.8 \mathrm{~Hz}, 2 \mathrm{H}), 7.83(\mathrm{~d}, J=9.1 \mathrm{~Hz}, 2 \mathrm{H}), 7.58(\mathrm{~d}, J=$ $8.3 \mathrm{~Hz}, 2 \mathrm{H}), 7.32(\mathrm{~d}, J=7.8 \mathrm{~Hz}, 2 \mathrm{H}), 2.41(\mathrm{~s}, 3 \mathrm{H})$.

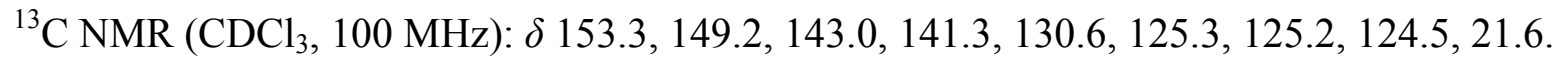

IR (powder): $v 3097,1527,1342,1047,851,722 \mathrm{~cm}^{-1}$.

$\mathrm{MS}\left(\mathrm{CI} / \mathrm{NH}_{3}\right): m / z 279\left(\mathrm{MNH}_{4}{ }^{+}\right), 262\left(\mathrm{MH}^{+}\right)$.

HRMS $m / z$ calculated for $\mathrm{C}_{13} \mathrm{H}_{11} \mathrm{NO}_{3} \mathrm{~S}\left(\mathrm{M}^{+}\right): 261.0460$. Found: 261.0441 .

Anal. Calcd for $\mathrm{C}_{13} \mathrm{H}_{11} \mathrm{NO}_{3} \mathrm{~S}: \mathrm{C}, 59.76 ; \mathrm{H}, 4.24 ; \mathrm{N}$, 5.36. Found: C, 59.77; H, 4.22; N, 5.25.

M.p. $119-121^{\circ} \mathrm{C}$.

p-trifluoromethylphenyl-p-tolyl sulfoxide 8a:<smiles>Cc1ccc(S(=O)c2ccc(C(F)(F)F)cc2)cc1</smiles>

Purification by silica gel flash chromatography (cyclohexane/ ethyl acetate, 9:1 $\rightarrow$ 85:15) to give a pale yellow oil ( $96 \%$ yield).

${ }^{1} \mathrm{H}$ NMR $\left(\mathrm{CDCl}_{3}, 400 \mathrm{MHz}\right): \delta 7.77(\mathrm{~d}, J=8.6 \mathrm{~Hz}, 2 \mathrm{H}), 7.71(\mathrm{~d}, J=8.4 \mathrm{~Hz}, 2 \mathrm{H}), 7.56(\mathrm{~d}, J=$ $8.4 \mathrm{~Hz}, 2 \mathrm{H}), 7.29$ (d, $J=7.8 \mathrm{~Hz}, 2 \mathrm{H}), 2.38$ (s, 3H).

${ }^{13} \mathrm{C} \mathrm{NMR}\left(\mathrm{CDCl}_{3}, 100 \mathrm{MHz}\right): \delta 150.3\left(\mathrm{C}_{\mathrm{Ar}}\right), 142.5\left(\mathrm{C}_{\mathrm{Ar}}\right), 141.9\left(\mathrm{C}_{\mathrm{Ar}}\right), 133.5\left(\mathrm{C}_{\mathrm{Ar}} / \mathrm{CF}_{3}\right), 133.1$ $\left(\mathrm{C}_{\mathrm{Ar}} / \mathrm{CF}_{3}\right), 132.9\left(\mathrm{C}_{\mathrm{Ar}} / \mathrm{CF}_{3}\right), 132.6\left(\mathrm{C}_{\mathrm{Ar}} / \mathrm{CF}_{3}\right), 130.4\left(\mathrm{CH}_{\mathrm{Ar}}\right), 127.6\left(\mathrm{C}_{\mathrm{Ar}} / \mathrm{CF}_{3}\right), 127.0\left(\mathrm{C}_{\mathrm{Ar}} / \mathrm{CF}_{3}\right)$, 126.3 and $126.2\left(\mathrm{CH}_{\mathrm{Ar}}\right), 125.4\left(\mathrm{C}_{\mathrm{Ar}} / \mathrm{CF}_{3}\right), 125.2\left(\mathrm{CH}_{\mathrm{Ar}}\right), 124.9\left(\mathrm{CH}_{\mathrm{Ar}}\right), 122.2\left(\mathrm{C}_{\mathrm{Ar}} / \mathrm{CF}_{3}\right), 21.5$. IR (neat): $v$ 2924, 1321, 1127, 1056, 808, $698 \mathrm{~cm}^{-1}$.

HRMS $m / z$ calculated for $\mathrm{C}_{14} \mathrm{H}_{11} \mathrm{OF}_{3} \mathrm{~S}\left(\mathrm{M}^{+}\right): 284.0483$. Found: 284.0463 .

\section{2-thiophenyl-p-tolyl sulfoxide 9a:}<smiles>Cc1ccc(S(=O)c2cccs2)cc1</smiles>

Purification by silica gel flash chromatography (cyclohexane/ethyl acetate, 9:1) to afford a brown oil ( $82 \%$ yield $)$. 
${ }^{1} \mathrm{H} \mathrm{NMR}\left(\mathrm{CDCl}_{3}, 400 \mathrm{MHz}\right): \delta$ 7.61-7.55 (m, 4H), $7.32(\mathrm{~d}, J=8.1 \mathrm{~Hz}, 2 \mathrm{H}), 7.06(\mathrm{dd}, J=5.1$, $3.8 \mathrm{~Hz}, 1 \mathrm{H}), 2.42(\mathrm{~s}, 3 \mathrm{H})$.

${ }^{13} \mathrm{C} \mathrm{NMR}\left(\mathrm{CDCl}_{3}, 100 \mathrm{MHz}\right): \delta$ 148.5, 142.0, 141.9, 132.3, 131.2, 130.0, 127.4, 124.5, 21.6.

IR (neat): $v 2921,1402,1082,1042,808,712 \mathrm{~cm}^{-1}$.

HRMS $m / z$ calculated for $\mathrm{C}_{11} \mathrm{H}_{10} \mathrm{OS}_{2}\left(\mathrm{M}^{+}\right)$: 222.0173. Found: 222.0183 .

p-bromophenyl-p-tolyl sulfoxide 10a:<smiles>Cc1ccc(S(=O)c2ccc(Br)cc2)cc1</smiles>

Purification by silica gel flash chromatography (cyclohexane/ethyl acetate 9:1 $\rightarrow$ 85:15) to afford the product as a brown solid (61\% yield).

${ }^{1} \mathrm{H} \mathrm{NMR}\left(\mathrm{CDCl}_{3}, 400 \mathrm{MHz}\right): \delta$ 7.60-7.57 (m, 2H), 7.53-7.48 (m, 4H), $7.27(\mathrm{~d}, J=8.1 \mathrm{~Hz}$, 2H), $2.37(\mathrm{~s}, 3 \mathrm{H})$.

${ }^{13} \mathrm{C} \mathrm{NMR}\left(\mathrm{CDCl}_{3}, 100 \mathrm{MHz}\right): \delta$ 145.1, 142.1, 142.0, 132.5, 130.3, 126.2, 125.4, 125.0, 21.5.

IR (powder): $v 2921,1471,1089,1048,1006,821,805,722 \mathrm{~cm}^{-1}$.

MS (CI/NH $): m / z 591,314\left(\mathrm{MNH}_{4}^{+}\right), 297\left(\mathrm{MH}^{+}\right)$.

HRMS $m / z$ calculated for $\mathrm{C}_{13} \mathrm{H}_{11}{ }^{79} \mathrm{BrOS}\left(\mathrm{M}^{+}\right)$: 293.9714. Found: 293.9731 .

M.p. $60-62^{\circ} \mathrm{C}$.

\section{1,2-(bis-p-tolylsulfinyl)benzene 11a:}

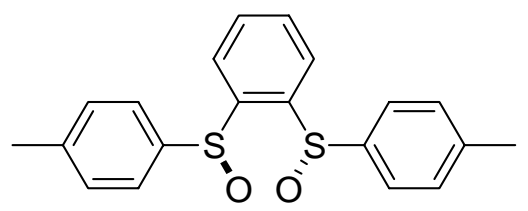

The reaction was performed with 0.5 equiv. of 1,2-diiodobenzene for $16 \mathrm{~h}$.

Purification by silica gel flash chromatography (cyclohexane/ethyl acetate, 95:5 $\rightarrow$ 9:1 $\rightarrow$ $85: 15 \rightarrow 8: 2 \rightarrow 7: 3$ ) to afford a pale yellow solid in a quantitative yield. 
Spectral data in agreement with those already reported by Tokunoh, R.; Sodeoka, M.; Aoe, K. I.; Shibasaki, M. Tetrahedron Lett. 1995, 36, 8035-8038.

${ }^{1} \mathrm{H} \mathrm{NMR}\left(\mathrm{CDCl}_{3}, 400 \mathrm{MHz}\right): \delta 8.02-7.98(\mathrm{~m}, 2 \mathrm{H}), 7.65(\mathrm{~d}, J=8.3 \mathrm{~Hz}, 2 \mathrm{H}), 7.62(\mathrm{dd}, J=5.8$, $3.3 \mathrm{~Hz}, 4 \mathrm{H}), 7.30$ (d, $J=7.8 \mathrm{~Hz}, 4 \mathrm{H}), 2.40$ (s, 6H).

${ }^{13} \mathrm{C} \mathrm{NMR}\left(\mathrm{CDCl}_{3}, 100 \mathrm{MHz}\right): \delta 143.9,142.6,140.9,132.4,130.5,125.8,124.5,21.6$.

IR (powder): $v$ 2922, 1433, 1397, 1082, 1055, 1008, 808, $697 \mathrm{~cm}^{-1}$.

(meso) 1,2-(bis-p-tolylsulfinyl)benzene 11a’:

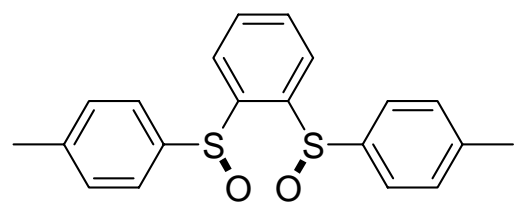

This product is obtained by treating 12 with wet neutral alumina and 2 equiv. of oxone ${ }^{\circledR}$ followed by purification by silica gel flash chromatography (cyclohexane/ethyl acetate, 9:1 $\rightarrow$ $8: 2 \rightarrow 7: 3)$ to give 11a' as a white solid (40\% overall yield, starting from 1a).

${ }^{1} \mathrm{H}$ NMR $\left(\mathrm{CDCl}_{3}, 400 \mathrm{MHz}\right): \delta$ 7.94-7.92 (m, 2H), 7.63-7.61 (m, 2H), $7.23(\mathrm{~d}, J=8.1 \mathrm{~Hz}$, 4H), 7.13 (d, $J=8.1 \mathrm{~Hz}, 4 \mathrm{H}), 2.32$ (s, 6H).

${ }^{13} \mathrm{C} \mathrm{NMR}\left(\mathrm{CDCl}_{3}, 100 \mathrm{MHz}\right): \delta 144.5,141.9,141.1,132.4,130.0,126.1,125.9,21.4$.

IR (powder): $v 2923,1445,1048,1026,803,756,620 \mathrm{~cm}^{-1}$.

MS (ESI $\left.{ }^{+}\right): m / z 747\left(2 \mathrm{M}+\mathrm{K}^{+}\right), 731\left(2 \mathrm{M}+\mathrm{Na}^{+}\right), 393\left(\mathrm{MK}^{+}\right), 377\left(\mathrm{MNa}^{+}\right), 355\left(\mathrm{MH}^{+}\right)$.

HRMS $m / z$ calculated for $\mathrm{C}_{20} \mathrm{H}_{18} \mathrm{O}_{2} \mathrm{~S}_{2}\left(\mathrm{M}^{+}\right)$: 355.0821 . Found: 355.0821 .

M.p. $123-125^{\circ} \mathrm{C}$.

(Z)-1-(hex-1-ene-1-sulfinyl)-4-methyl-benzene 15a:

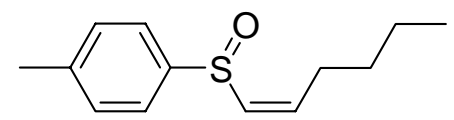

Purification by silica gel flash chromatography (ethyl acetate/cyclohexane, 9:1 $\rightarrow$ 85:15 $\rightarrow$ $8: 2)$ to afford the product as a colorless oil (33\% yield). 
Spectral data in agreement with those already reported by Maezaki, N.; Izumi, M.; Yuyama, S.; Sawamoto, H.; Iwata, C.; Tanaka, T. Tetrahedron 2000, 56, 7927-7945.

${ }^{1} \mathrm{H}$ NMR $\left(\mathrm{CDCl}_{3}, 400 \mathrm{MHz}\right): \delta 7.51(\mathrm{~d}, J=8.4 \mathrm{~Hz}, 2 \mathrm{H}), 7.32(\mathrm{~d}, J=7.8 \mathrm{~Hz}, 2 \mathrm{H}), 6.24-6.16$ (m, 2H), 2.68-2.61 (dt, $J=14.1,6.6 \mathrm{~Hz}, 1 \mathrm{H}), 2.58-2.51(\mathrm{dt}, J=14.1,6.6 \mathrm{~Hz}, 1 \mathrm{H}), 2.42$ (s, $3 \mathrm{H}), 1.51-1.40(\mathrm{~m}, 2 \mathrm{H}), 1.31-1.27(\mathrm{~m}, 2 \mathrm{H}), 0.96(\mathrm{t}, J=7.3 \mathrm{~Hz}, 3 \mathrm{H})$.

${ }^{13} \mathrm{C} \mathrm{NMR}\left(\mathrm{CDCl}_{3}, 100 \mathrm{MHz}\right): \delta 142.1,141.5,141.2,137.1,130.1,124.2,31.3,29.2,22.4$, $21.5,14.0$.

(V) Characterization data for aromatic sulfoxides starting from $1 \mathrm{~b}$

\section{2-naphthyl-p-tolyl sulfoxide 13b:}<smiles>Cc1ccc(S(=O)c2ccc3ccccc3c2)cc1</smiles>

Purification by silica gel flash chromatography (cyclohexane/ethyl acetate, 9:1 $\rightarrow$ 8:2) to afford a pale brown solid (79\% yield).

Spectral data in agreement with those already reported by Furukawa, N.; Ogawa, S.; Matsumura, K.; Fujihara, H. J. Org. Chem. 1991, 56, 6341-6348.

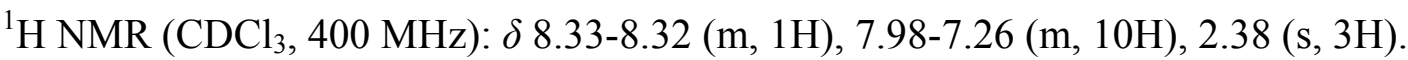
M.p. $82-84{ }^{\circ} \mathrm{C}$.

2-naphthyl-p-anisyl sulfoxide 14b:<smiles>COc1ccc(S(=O)c2ccc3ccccc3c2)cc1</smiles>

Purification by silica gel flash chromatography (cyclohexane/ethyl acetate, 9:1 $\rightarrow$ 8:2 $\rightarrow$ 7:3) to afford a pale brown solid ( $45 \%$ yield).

${ }^{1} \mathrm{H}$ NMR $\left(\mathrm{CDCl}_{3}, 400 \mathrm{MHz}\right): \delta 8.33$ (s, 1H), 7.98-7.95 (m, 1H), 7.88-7.85 (m, 2H), 7.64-7.57 (m, 4H), 7.46-7.43 (m, 1H), $6.96(\mathrm{~d}, J=8.8 \mathrm{~Hz}, 2 \mathrm{H}), 3.82(\mathrm{~s}, 3 \mathrm{H})$. 
${ }^{13} \mathrm{C} \mathrm{NMR}\left(\mathrm{CDCl}_{3}, 100 \mathrm{MHz}\right): \delta 162.2,142.8,136.6,134.3,132.9,129.6,128.7,128.1,127.9$, $127.6,127.3,125.0,120.8,115.0,55.6$.

IR (powder): $v 2964,1592,1494,1252,1039,816 \mathrm{~cm}^{-1}$.

$\mathrm{MS}\left(\mathrm{CI} / \mathrm{NH}_{3}\right): m / z 300\left(\mathrm{MNH}_{4}{ }^{+}\right), 283\left(\mathrm{M}^{+}\right)$.

HRMS $m / z$ calculated for $\mathrm{C}_{17} \mathrm{H}_{14} \mathrm{O}_{2} \mathrm{~S}\left(\mathrm{M}^{+}\right)$: 282.0715. Found: 282.0719 .

M.p. $92-94{ }^{\circ} \mathrm{C}$.

(VI) Characterization data for aromatic sulfoxides starting from 1c

\section{benzyl-p-tolyl sulfoxide 13c:}

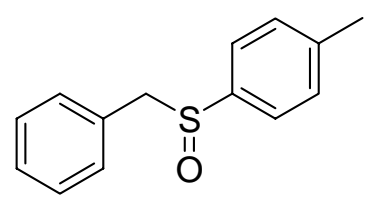

Purification by silica gel flash chromatography (cyclohexane/ethyl acetate, 9:1 $\rightarrow$ 85:15 $\rightarrow$ $8: 2 \rightarrow 7: 3)$ followed by recrystallization from $n$-hexane/toluene $(2: 1)$ to afford colourless needles (70\% yield).

Spectral data in agreement with those already reported by Katritzky, A. R.; Yang, B.; Qian, Y. Synlett 1996, 701702.

${ }^{1} \mathrm{H}$ NMR $\left(\mathrm{CDCl}_{3}, 400 \mathrm{MHz}\right): \delta$ 7.33-7.23 (m, 7H), 7.02-6.99 (m, 2H), $4.05(\mathrm{AB}, J=12.4 \mathrm{~Hz}$, $2 \mathrm{H}), 2.41(\mathrm{~s}, 3 \mathrm{H})$.

${ }^{13} \mathrm{C} \mathrm{NMR}\left(\mathrm{CDCl}_{3}, 100 \mathrm{MHz}\right): \delta 142.8,141.7,130.4,129.6,128.9,128.5,128.3,124.5,63.8$, 21.5 .

benzyl-p-anisyl sulfoxide 14c:<smiles>COc1ccc(S(=O)Cc2ccccc2)cc1</smiles> 
Purification by silica gel flash chromatography (cyclohexane/ethyl acetate, 95:5 $\rightarrow$ 9:1 $\rightarrow$ $85: 15 \rightarrow 8: 2 \rightarrow 7: 3$ ) to afford the product as a pale brown solid ( $46 \%$ yield).

Spectral data in agreement with those already reported by Donnoli, M. I.; Superchi, S.; Rosini, C. J. Org. Chem. 1998, 63, 9392-9395.

${ }^{1} \mathrm{H} \mathrm{NMR}\left(\mathrm{CDCl}_{3}, 400 \mathrm{MHz}\right): \delta 7.33-7.24(\mathrm{~m}, 5 \mathrm{H}), 7.01-6.92(\mathrm{~m}, 4 \mathrm{H}), 4.04(\mathrm{AB}, J=12.4 \mathrm{~Hz}$, $2 \mathrm{H}), 3.85$ (s, 3H).

${ }^{13} \mathrm{C} \mathrm{NMR}\left(\mathrm{CDCl}_{3}, 100 \mathrm{MHz}\right): \delta 162.1,133.7,130.5,129.4,128.6,128.3,126.5,114.4,63.8$, 55.6 .

(VII) Characterization data for aromatic sulfoxides starting from 1d

\section{isopropyl-p-tolyl sulfoxide 13d:}

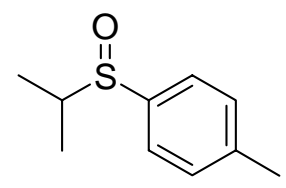

Purification by silica gel flash chromatography (cyclohexane/ethyl acetate, $8: 2 \rightarrow 7: 3$ ) to afford the product as a pale yellow oil ( $41 \%$ yield).

${ }^{1} \mathrm{H}$ NMR $\left(\mathrm{CDCl}_{3}, 400 \mathrm{MHz}\right): \delta 7.47(\mathrm{~d}, J=8.1 \mathrm{~Hz}, 2 \mathrm{H}), 7.31(\mathrm{~d}, J=8.1 \mathrm{~Hz}, 2 \mathrm{H}), 2.82$ (hept, $J=6.8 \mathrm{~Hz}, 1 \mathrm{H}), 2.42(\mathrm{~s}, 3 \mathrm{H}), 1.20(\mathrm{~d}, J=6.8 \mathrm{~Hz}, 3 \mathrm{H}), 1.15(\mathrm{~d}, J=6.8 \mathrm{~Hz}, 3 \mathrm{H})$.

${ }^{13} \mathrm{C} \mathrm{NMR}\left(\mathrm{CDCl}_{3}, 100 \mathrm{MHz}\right): \delta 141.5,138.5,129.7,125.1,54.6,21.5,15.8,14.2$.

IR (neat): $v 2925,1462,1085,1041,1022,811 \mathrm{~cm}^{-1}$.

HRMS $m / z$ calculated for $\mathrm{C}_{10} \mathrm{H}_{14} \mathrm{OS}\left(\mathrm{M}^{+}\right)$: 182.0765. Found: 182.0752 .

\section{isopropyl-p-anisyl sulfoxide $14 d$ :}<smiles>COc1ccc(S(=O)C(C)C)cc1</smiles> 
Purification by silica gel flash chromatography (cyclohexane/ethyl acetate, 8:2 $\rightarrow 75: 25$ ) to give the product as a pale brown oil (35\% yield).

${ }^{1} \mathrm{H} \mathrm{NMR}\left(\mathrm{CDCl}_{3}, 400 \mathrm{MHz}\right): \delta 7.54(\mathrm{~d}, J=8.8 \mathrm{~Hz}, 2 \mathrm{H}), 7.03(\mathrm{~d}, J=8.8 \mathrm{~Hz}, 2 \mathrm{H}), 3.87(\mathrm{~s}$, $3 \mathrm{H}), 2.82$ (hept, $J=6.8 \mathrm{~Hz}, 1 \mathrm{H}), 1.19$ (d, $J=6.8 \mathrm{~Hz}, 3 \mathrm{H}), 1.17$ (d, $J=6.8 \mathrm{~Hz}, 3 \mathrm{H})$.

${ }^{13} \mathrm{C} \mathrm{NMR}\left(\mathrm{CDCl}_{3}, 100 \mathrm{MHz}\right): \delta 162.1,132.7,127.0,114.5,55.6,54.7,15.6,14.6$.

IR (neat): $v 2925,1596,1496,1251,1025,738 \mathrm{~cm}^{-1}$.

HRMS $m / z$ calculated for $\mathrm{C}_{10} \mathrm{H}_{14} \mathrm{O}_{2} \mathrm{~S}\left(\mathrm{M}^{+}\right)$: 198.0715. Found: 198.0702 .

(VIII) Experimental procedure for the pseudo-domino arylation/Mizoroki-Heck sequence and characterization data for compound 16

To a solution of tris(dibenzylideneacetone)dipalladium (5 mol\%) in DMF (500 $\mu \mathrm{L})$ was added xantphos $(10 \mathrm{~mol} \%)$. The solution was stirred at room temperature for $5 \mathrm{~min}$. Then, a solution of $p$-iodo-bromobenzene ( $0.373 \mathrm{mmol}$ in $1.5 \mathrm{~mL}$ of DMF), 3-p-tolylsulfanyl-propionic acid tert-butyl ester 1a $(0.373 \mathrm{mmol}$ in $1.5 \mathrm{~mL}$ of $\mathrm{DMF})$, and $\mathrm{Cs}_{2} \mathrm{CO}_{3}(1.87 \mathrm{mmol}, 5$ equiv. $)$ were successively added. The resulting system was stirred vigorously at $130{ }^{\circ} \mathrm{C}$ for $72 \mathrm{~h}$. Then, after cooling to room temperature, water $(5 \mathrm{~mL})$ was added and the aqueous phase was extracted two times with dichloromethane. The collected organic layers were dried over anhydrous $\mathrm{MgSO}_{4}$ and the solvent was removed under reduced pressure. The crude material was purified by flash column chromatography (cyclohexane/ethyl acetate, 9:1 $\rightarrow$ 8:2 $\rightarrow$ 7:3) to afford 16 (41 mg, 32\% yield) as a white solid.

\section{(E)-3-[4-(tolyl-4-sulfinyl)-phenyl]-acrylic acid tert-butyl ester 16:}

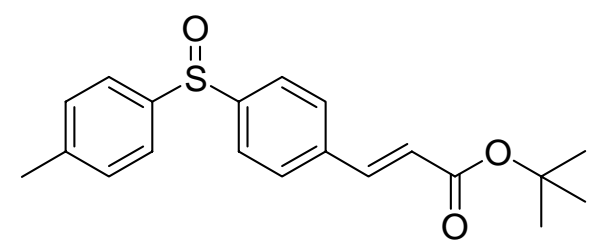

${ }^{1} \mathrm{H}$ NMR $\left(\mathrm{CDCl}_{3}, 400 \mathrm{MHz}\right): \delta$ 7.65-7.63 (m, 2H), 7.59-7.53 (m, 5H), $7.28(\mathrm{~d}, J=7.8 \mathrm{~Hz}$, 2H), $6.40(\mathrm{~d}, J=15.9 \mathrm{~Hz}, 1 \mathrm{H}), 2.38(\mathrm{~s}, 3 \mathrm{H}), 1.53(\mathrm{~s}, 9 \mathrm{H})$.

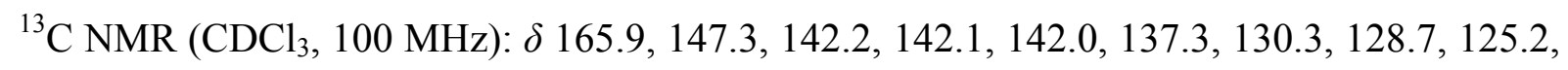
$125.1,122.3,81.0,28.2,21.5$. 
IR (powder): $v$ 2977, 1704, 1635, 1320, 1144, 1045, $804 \mathrm{~cm}^{-1}$. $\operatorname{MS}\left(\mathrm{CI} / \mathrm{NH}_{3}\right): m / z 343\left(\mathrm{MH}^{+}\right)$.

HRMS $m / z$ calculated for $\mathrm{C}_{20} \mathrm{H}_{22} \mathrm{O}_{3} \mathrm{~S}\left(\mathrm{M}^{+}\right)$: 342.1290. Found: 342.1289. M.p. $82-84{ }^{\circ} \mathrm{C}$. 
3-benzylsulfanyl-propionic acid tert-butyl ester

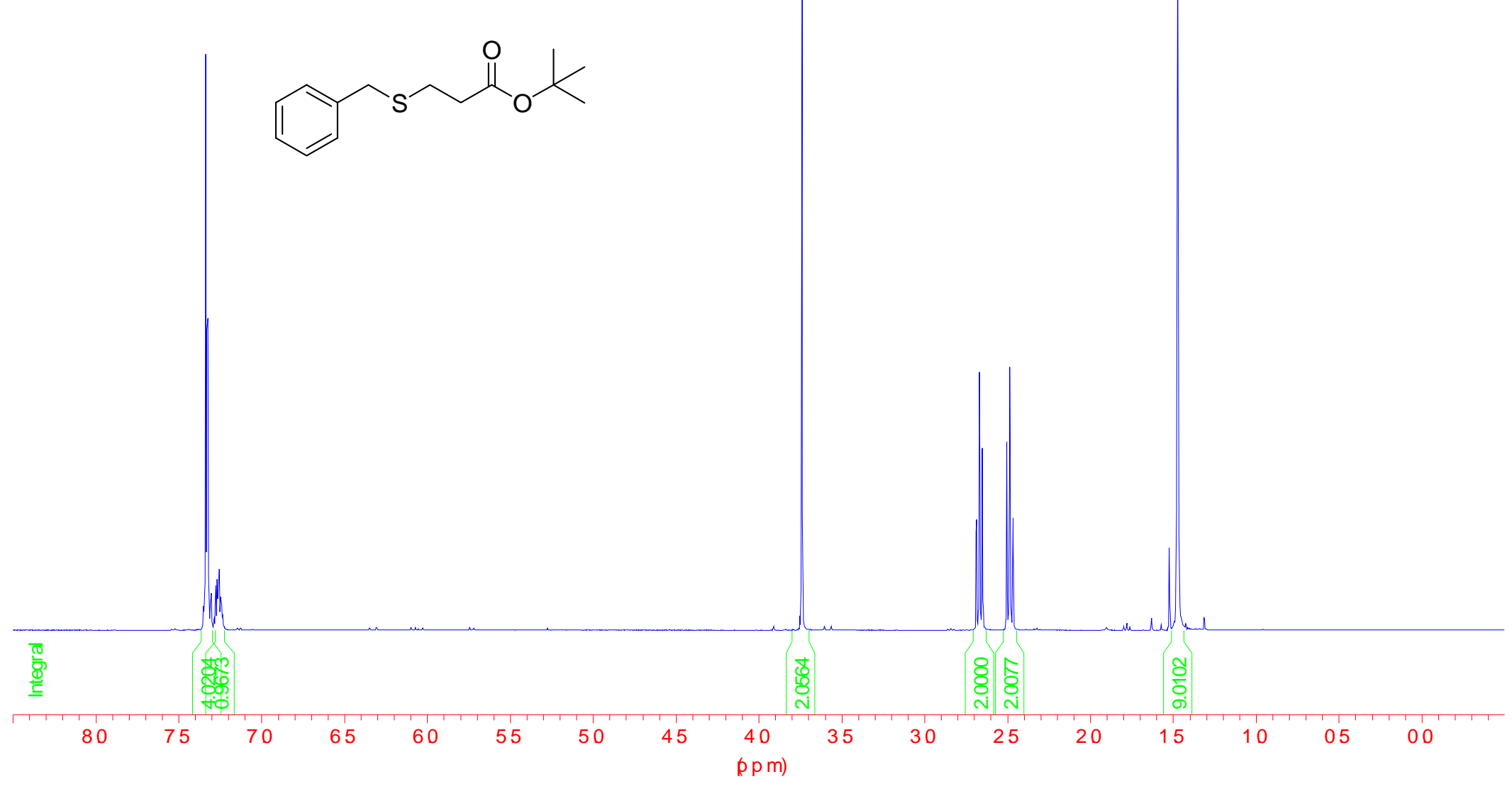


3-benzylsulfanyl-propionic acid tert-butyl ester

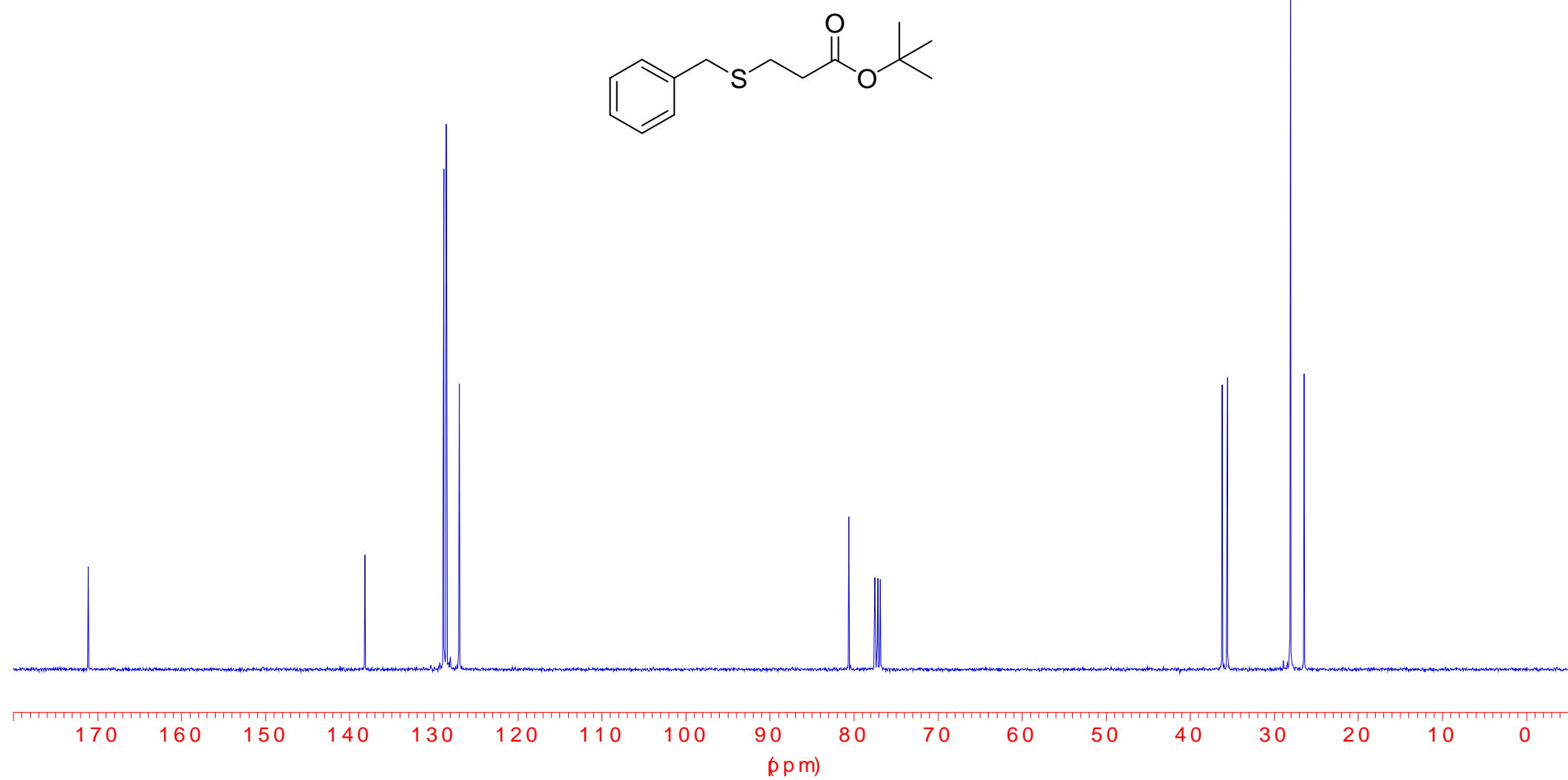




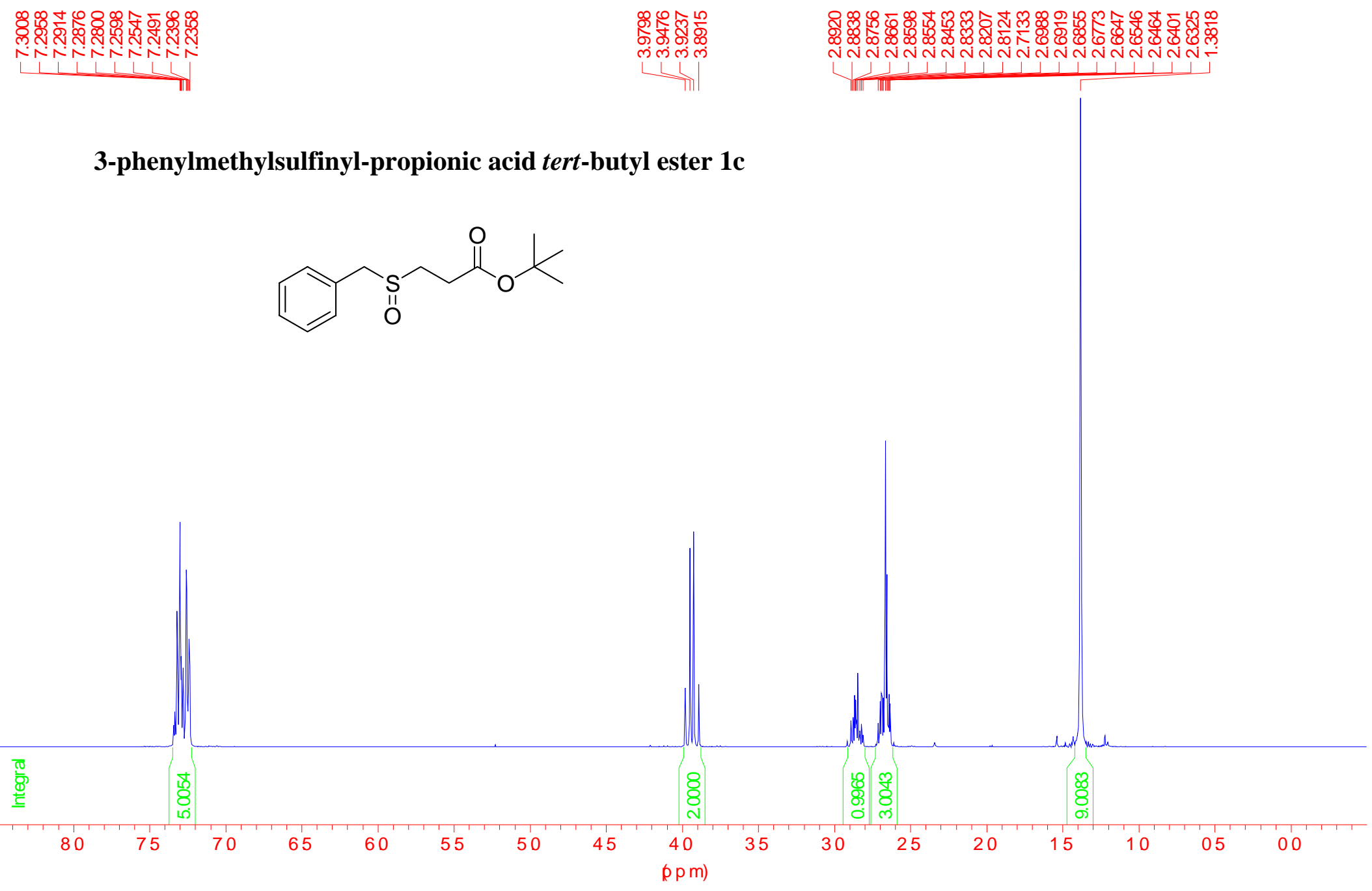




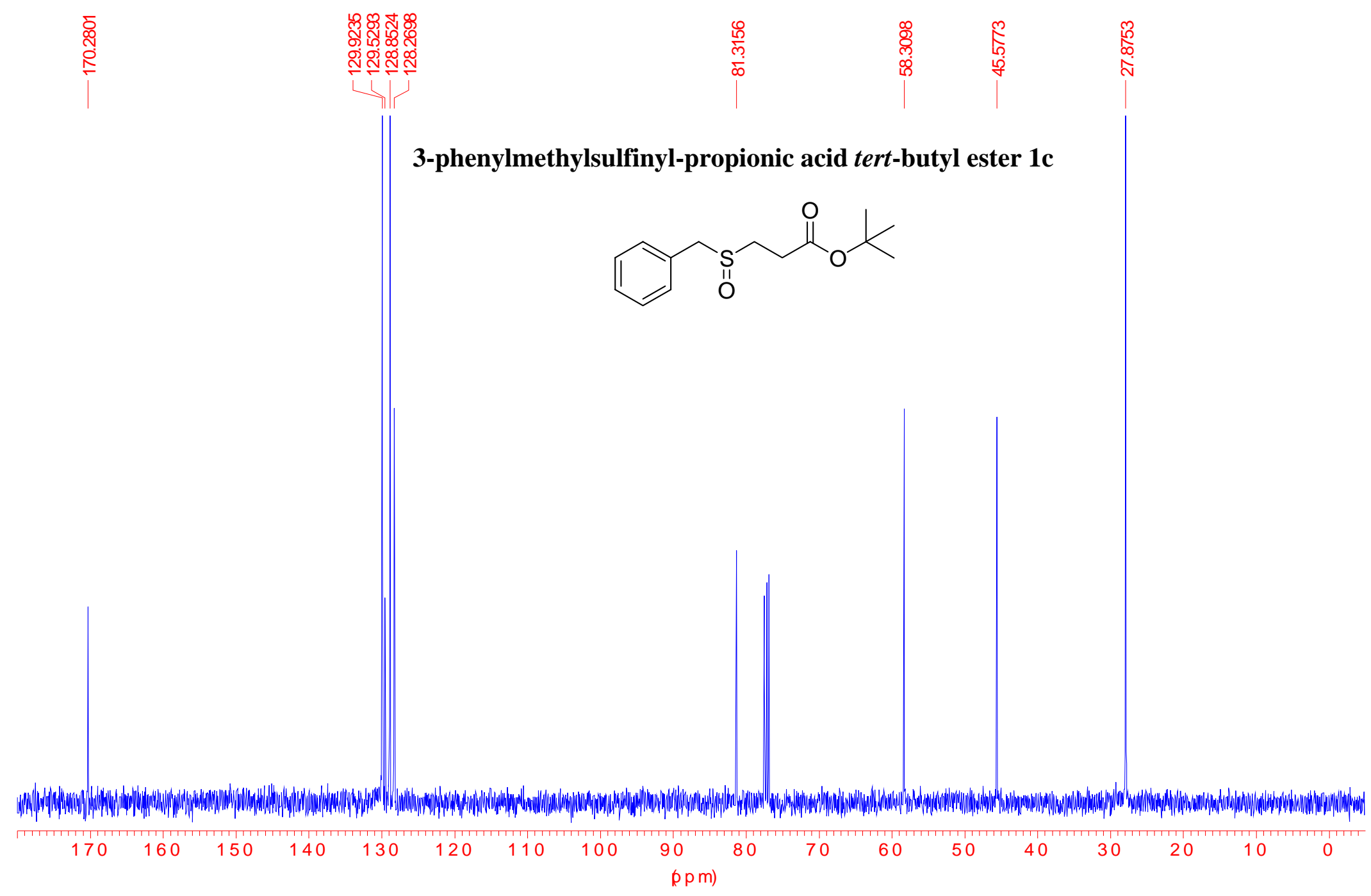




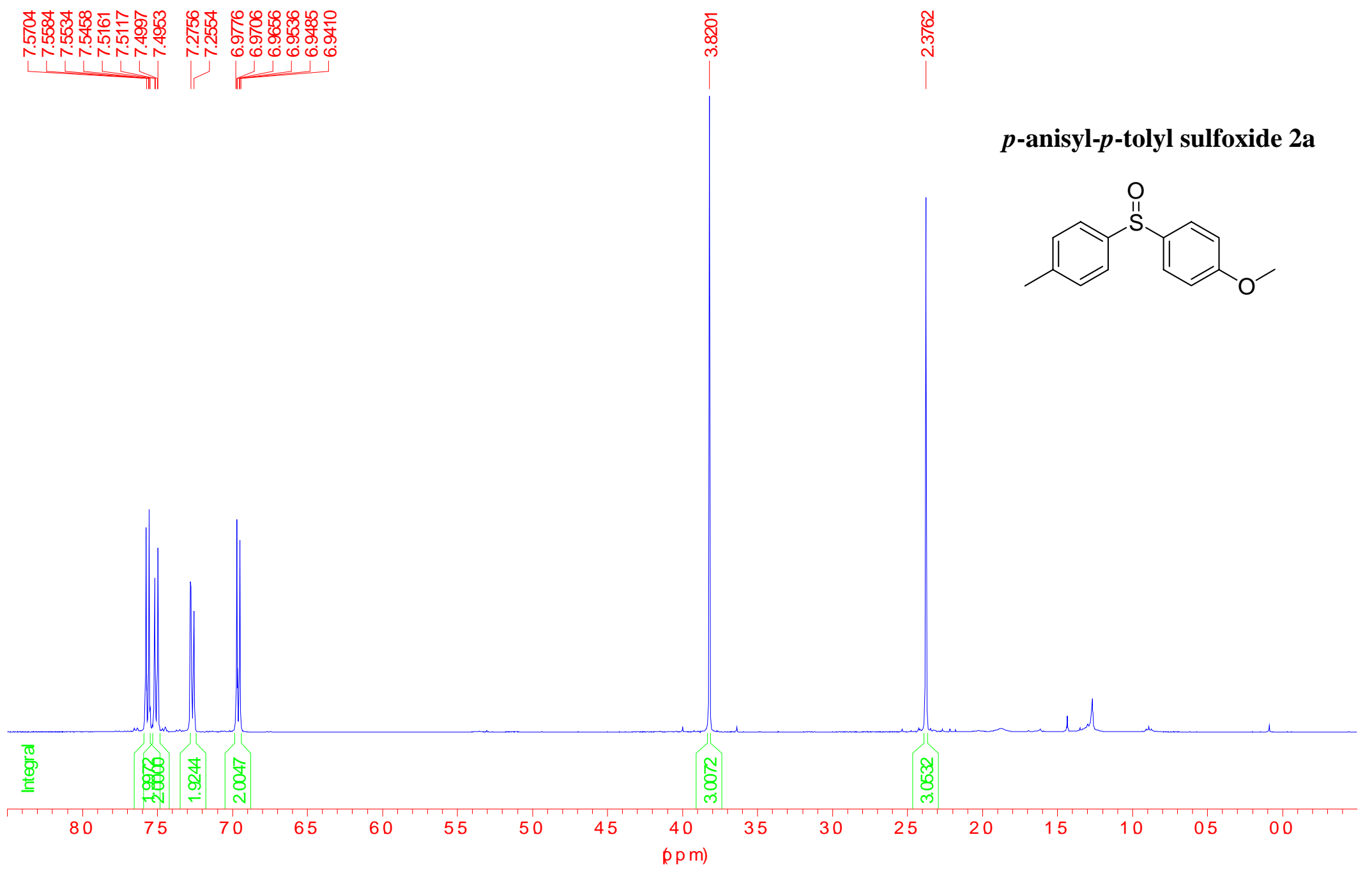




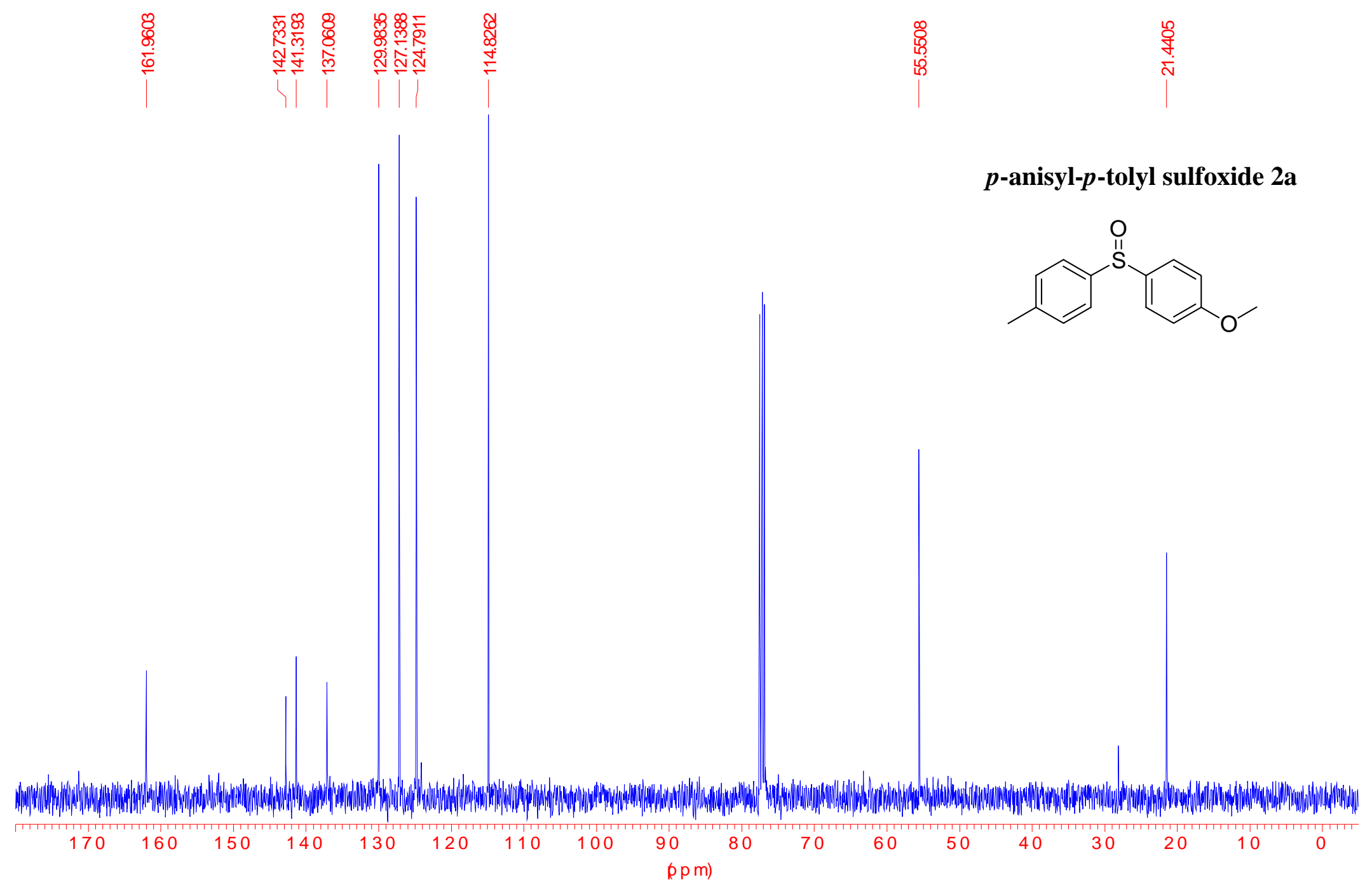




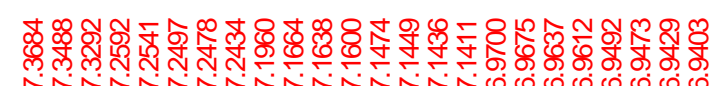

丞

m-anisyl-p-tolyl sulfoxide $4 a$

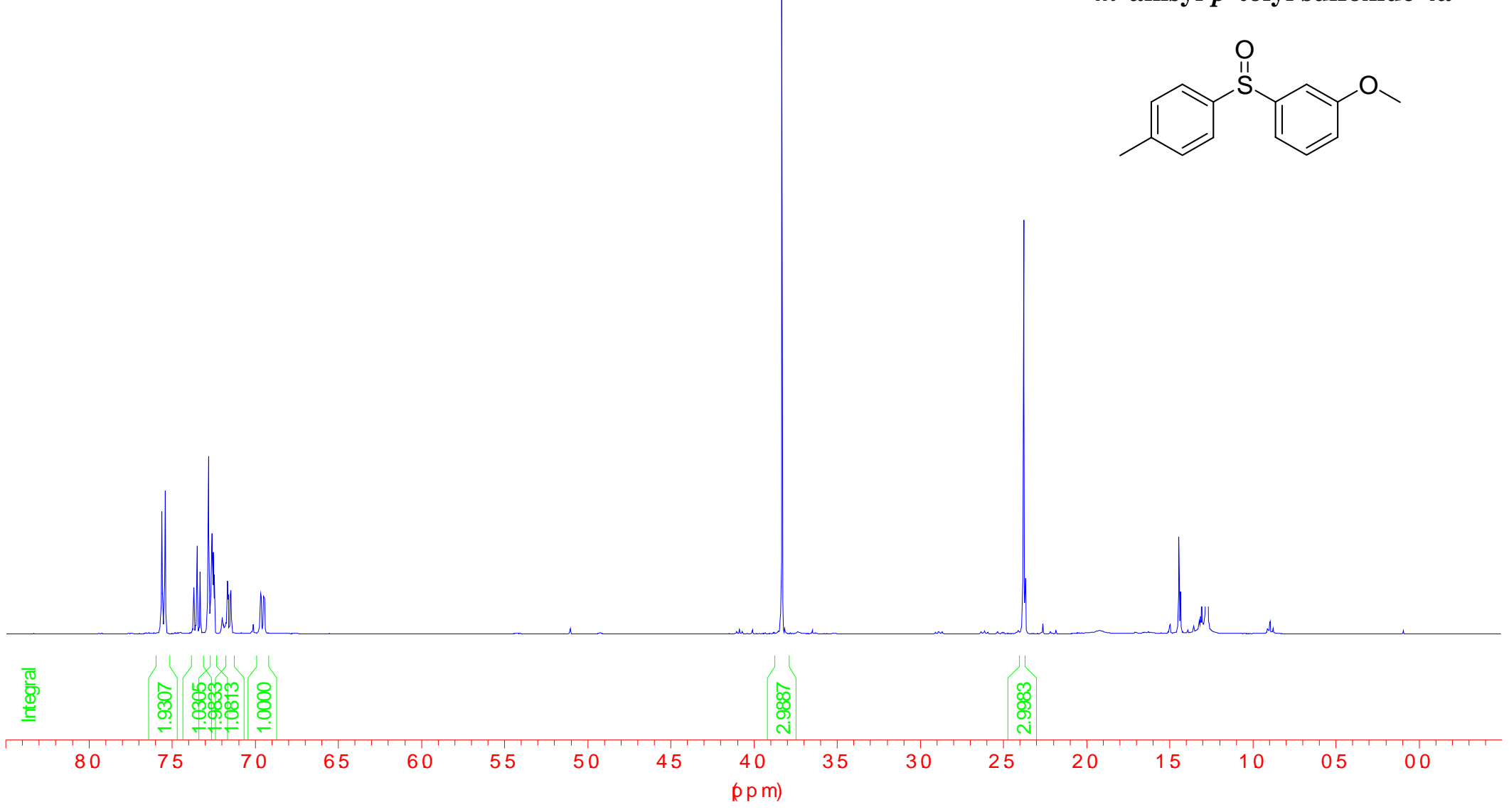




\section{m-anisyl-p-tolyl sulfoxide 4a}
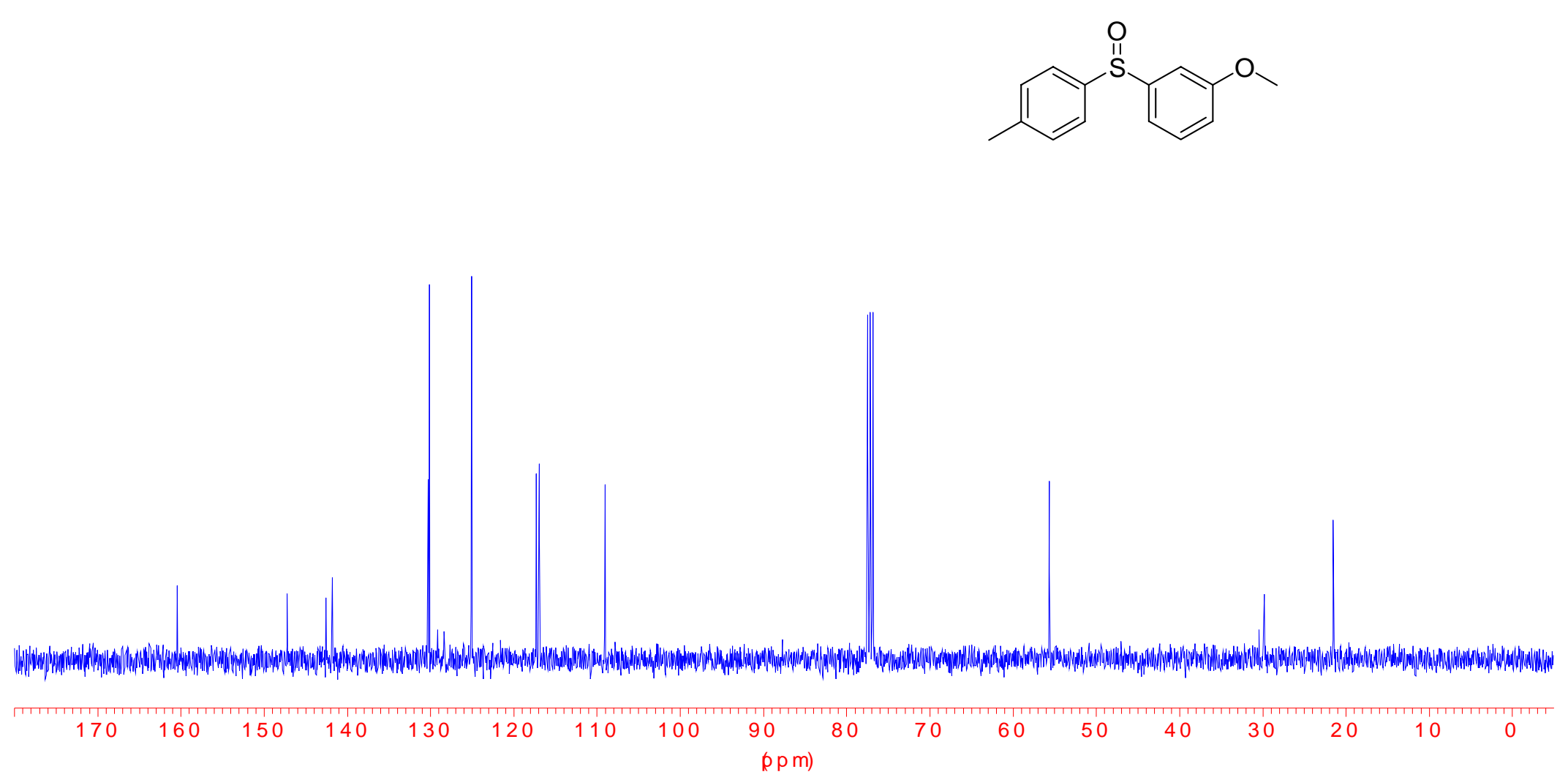


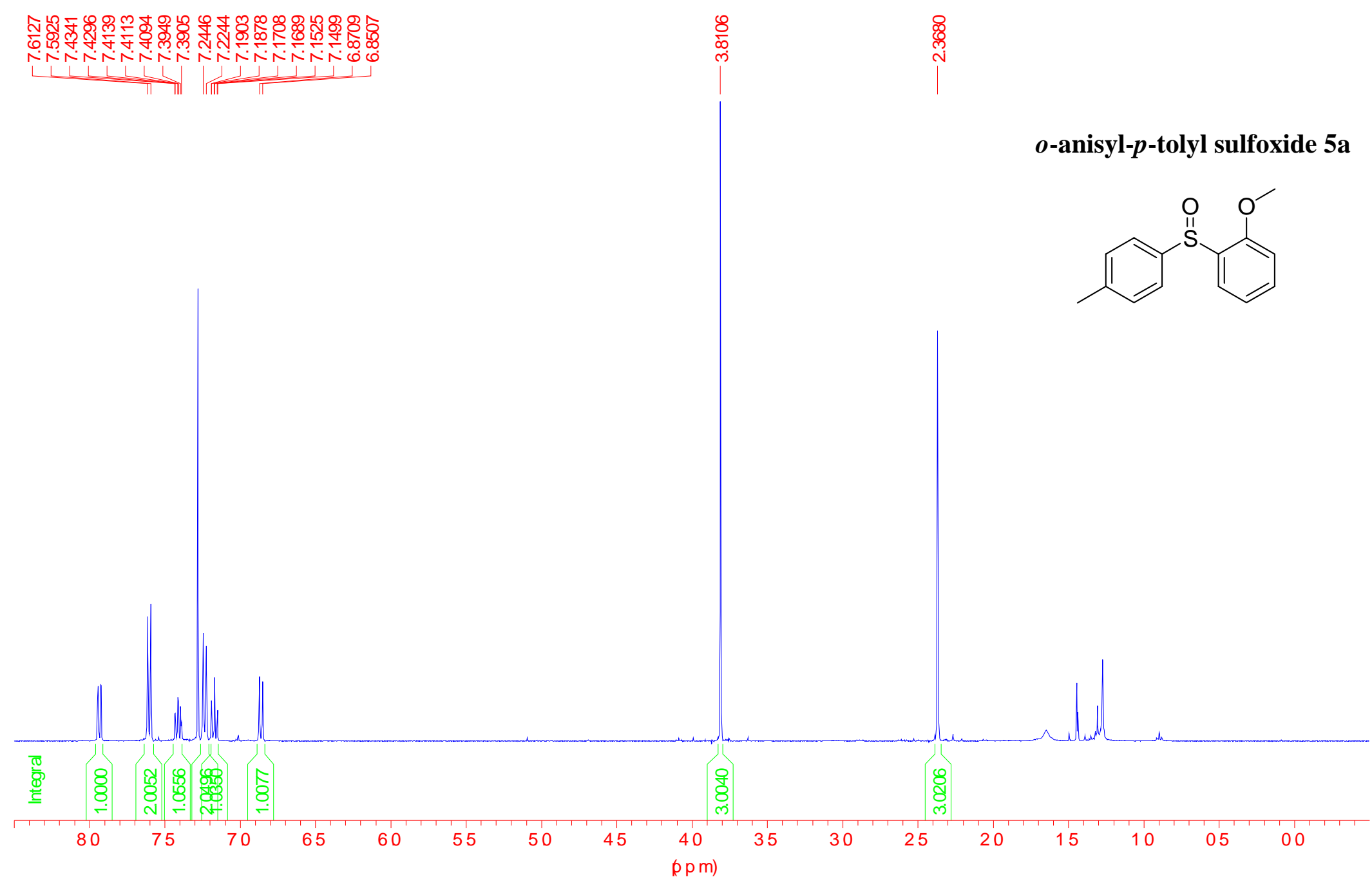




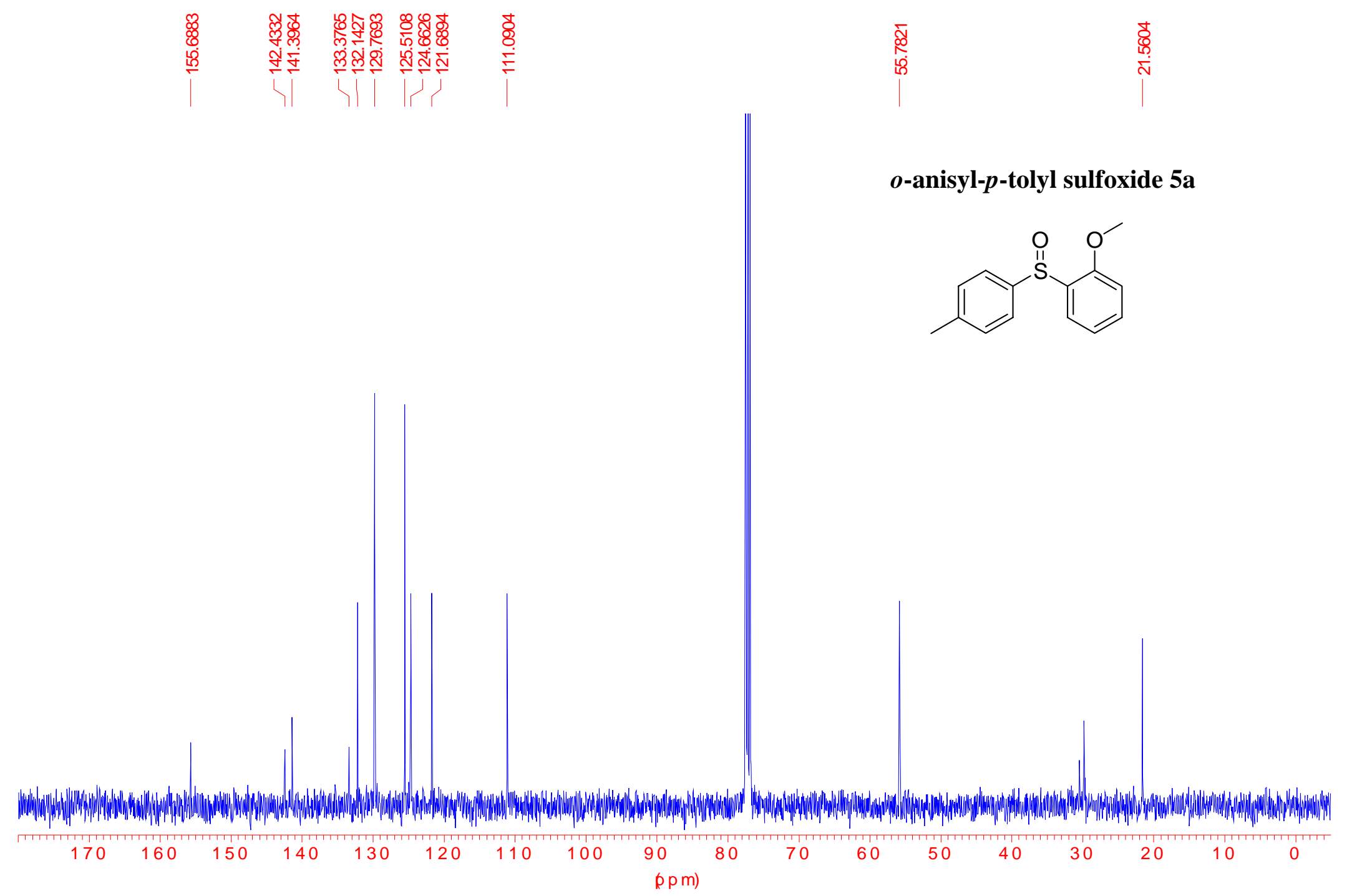




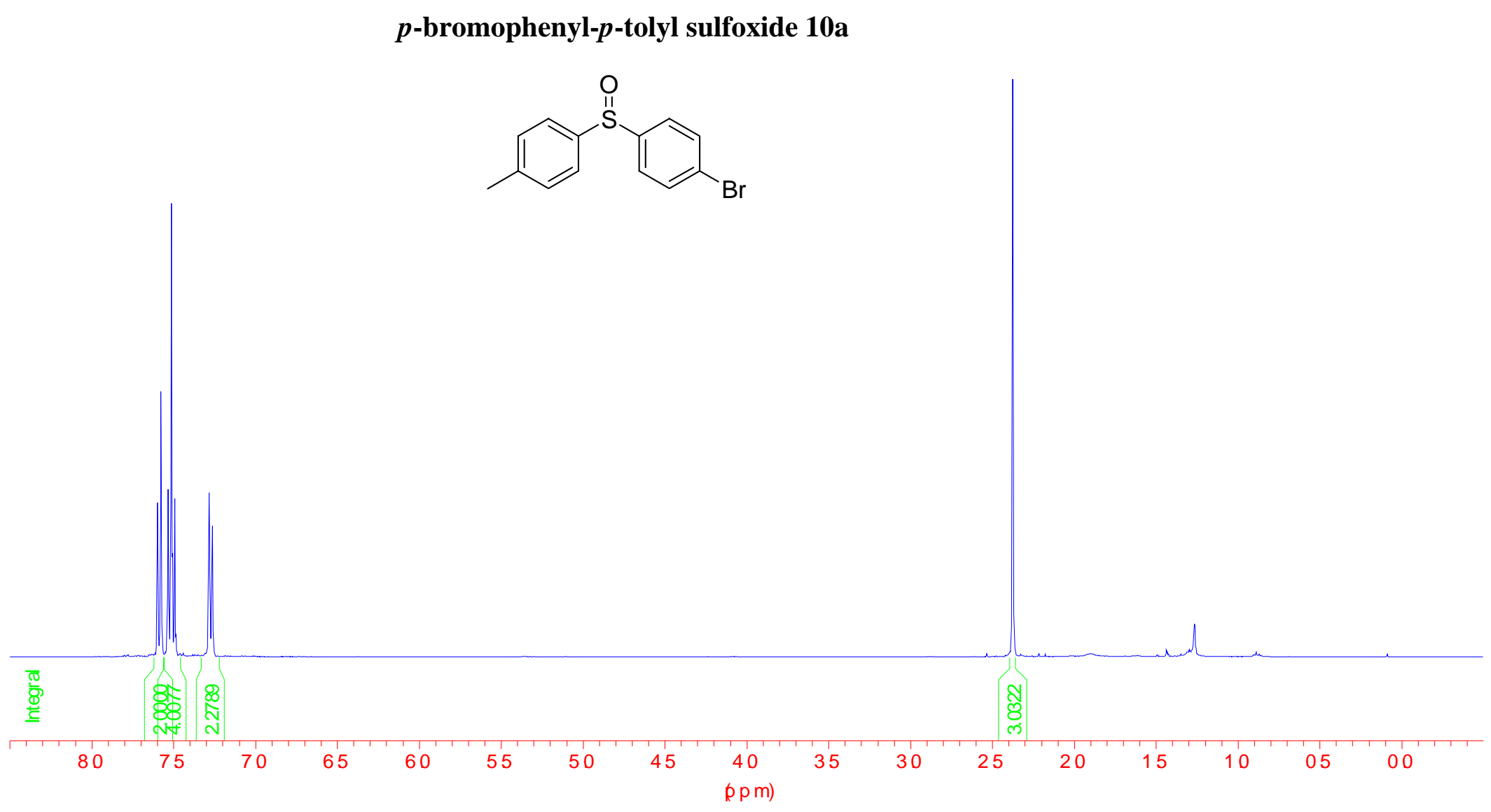




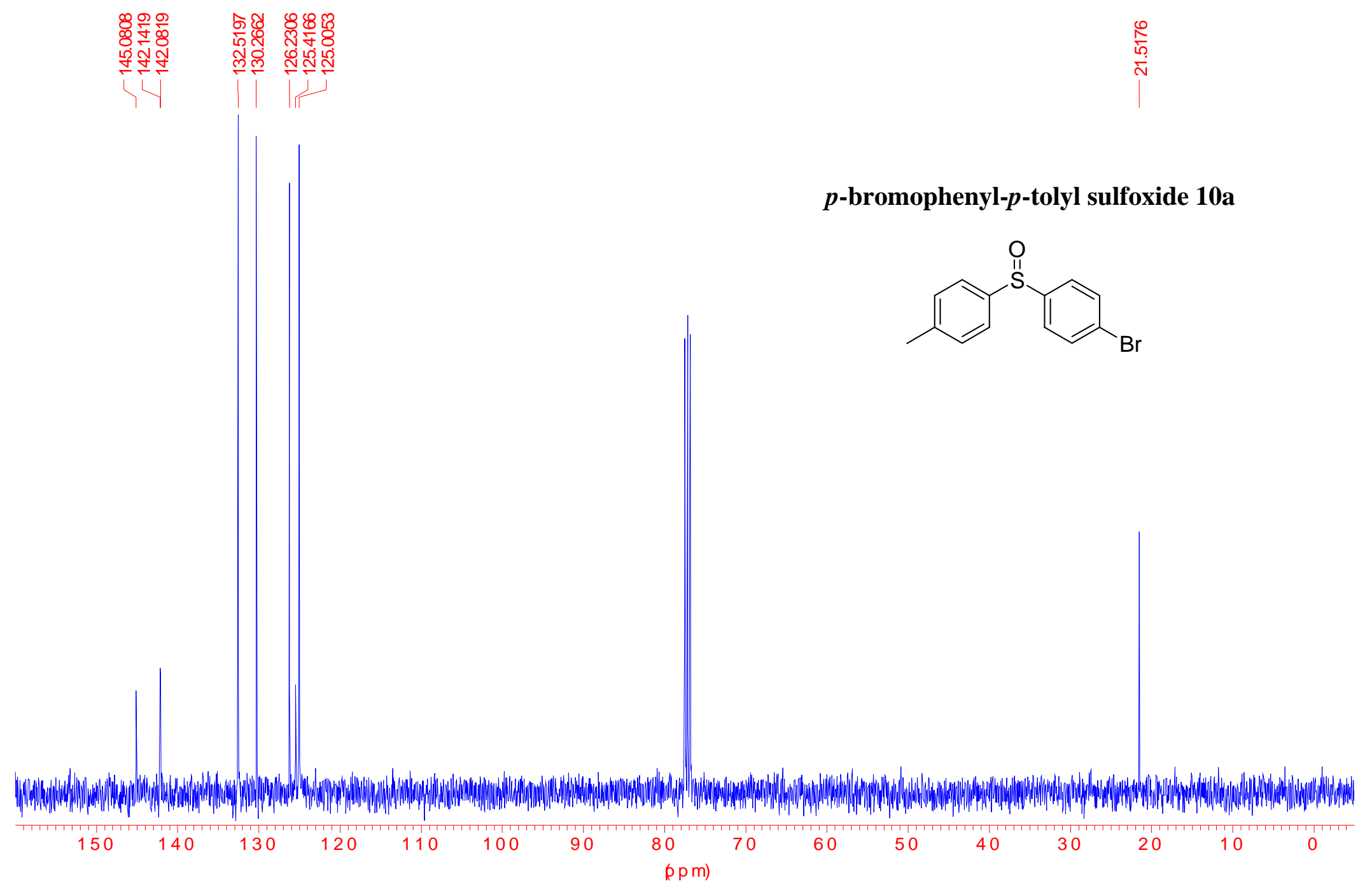




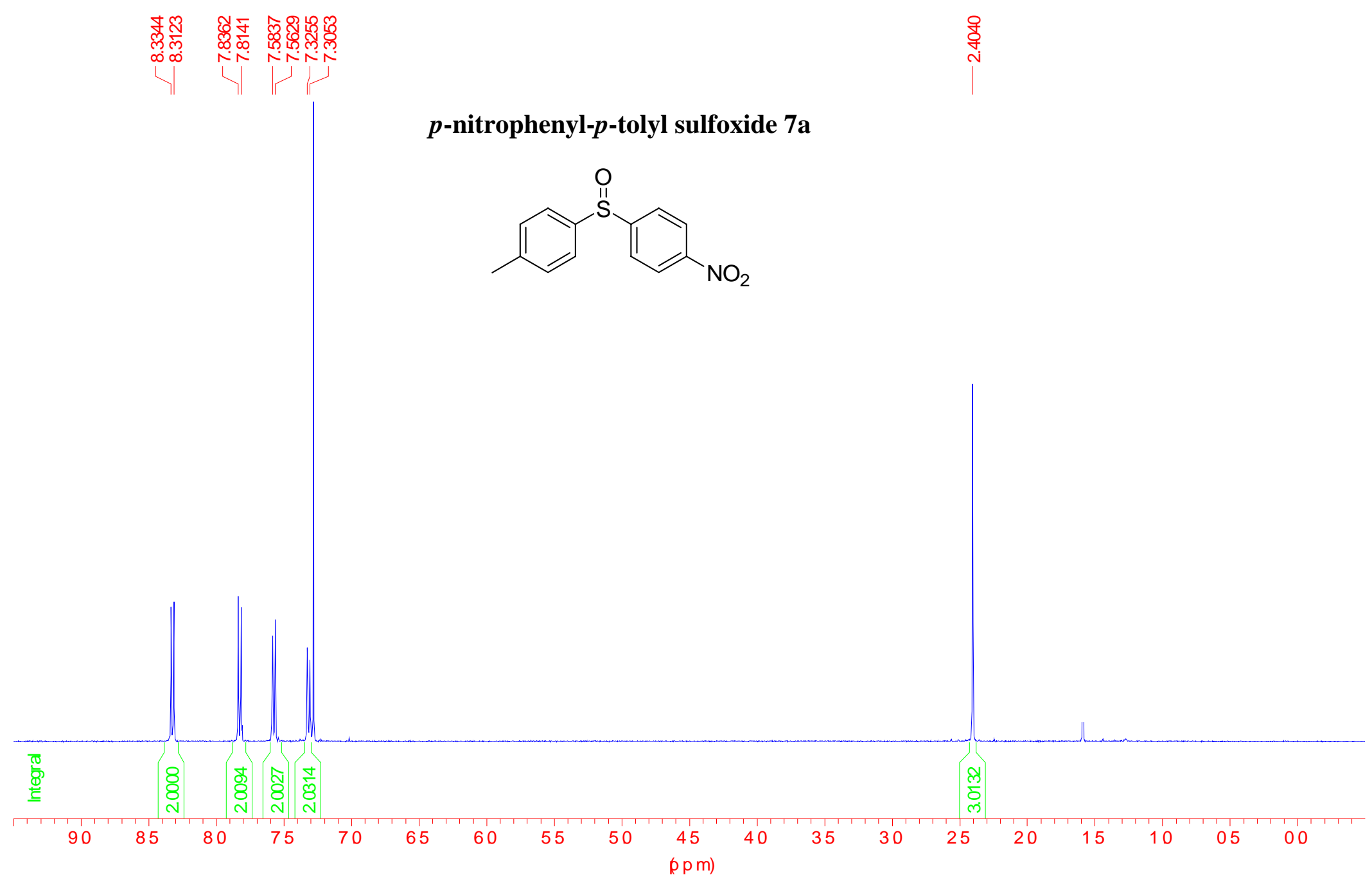




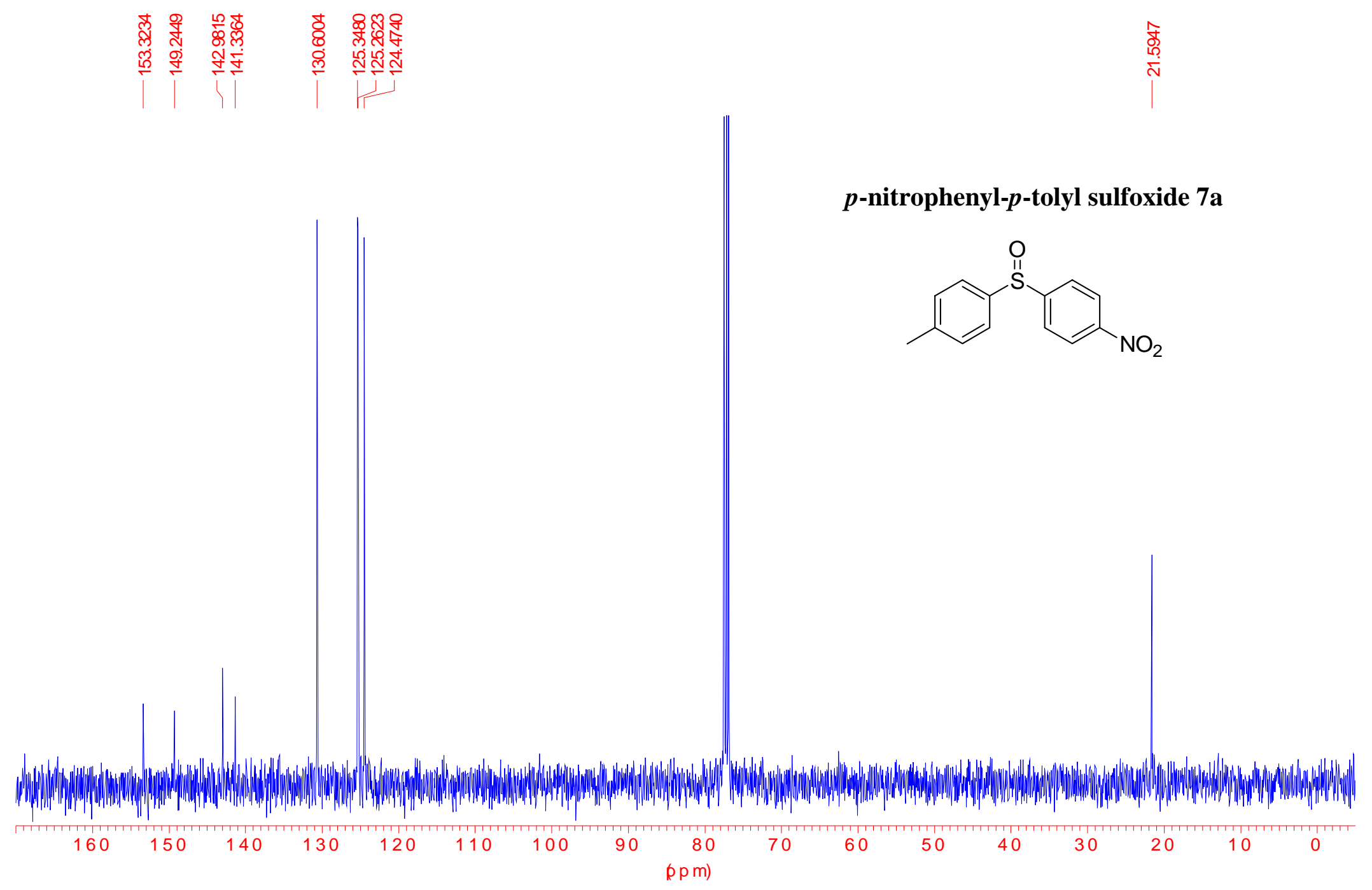




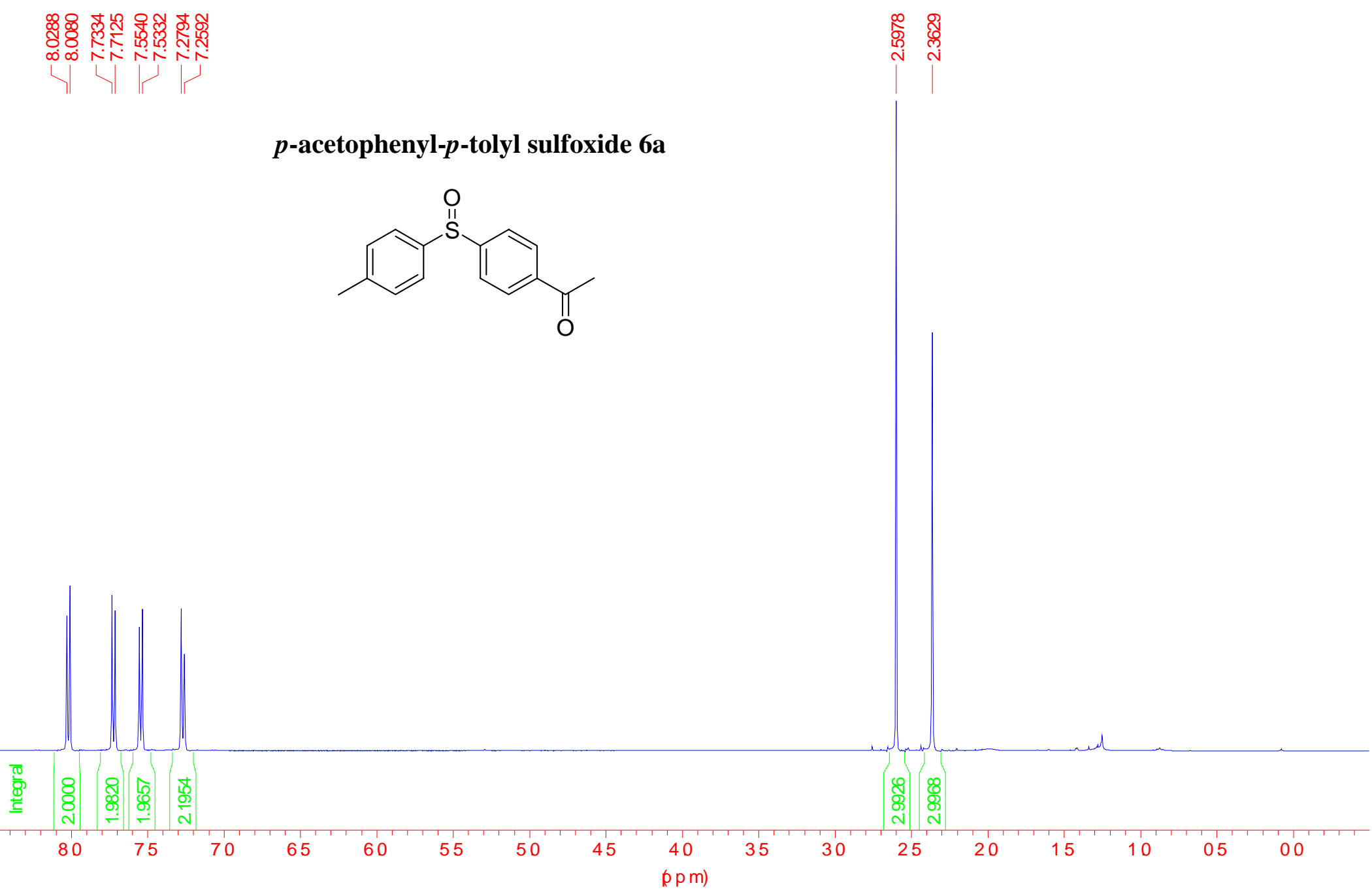




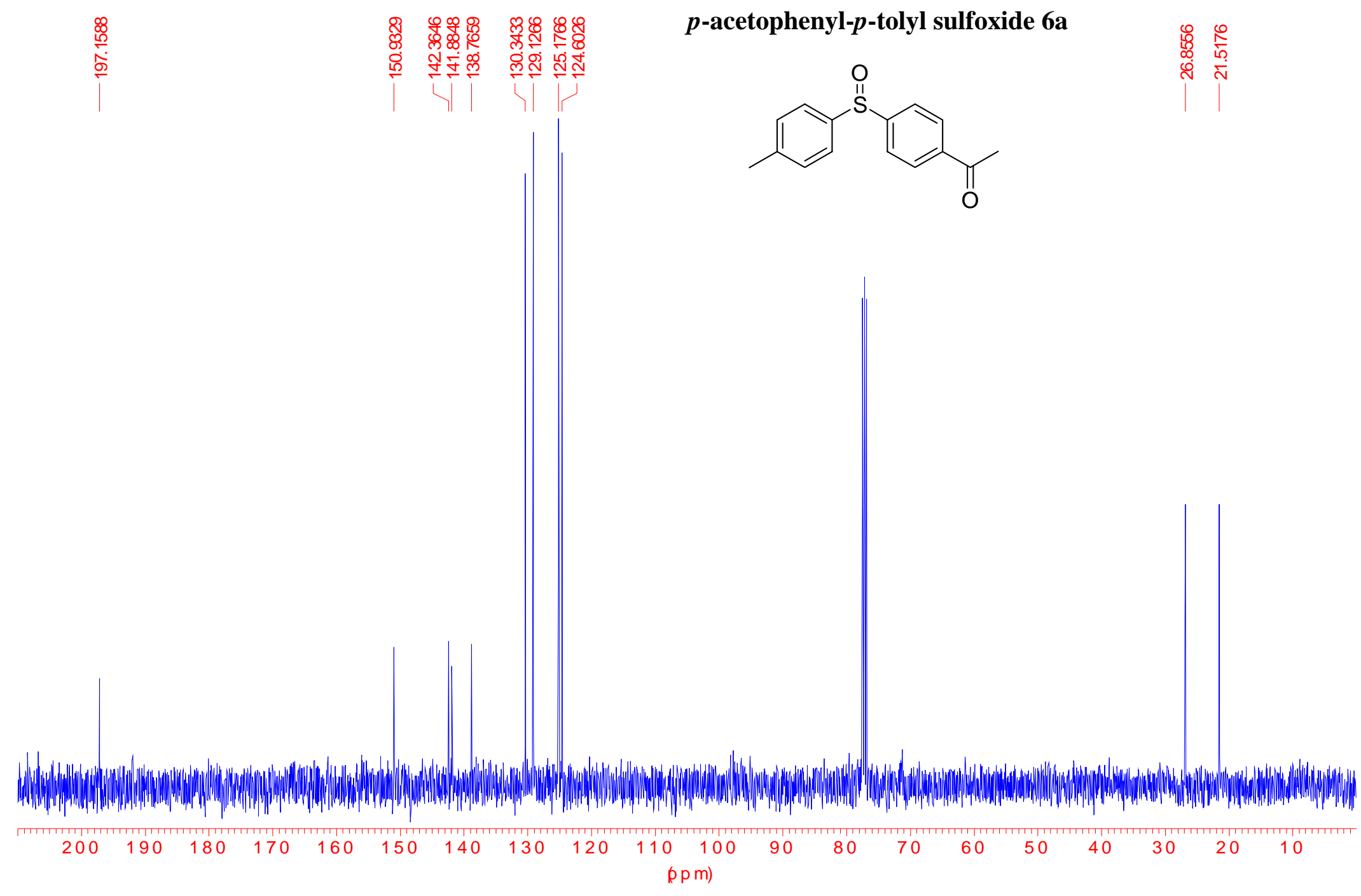




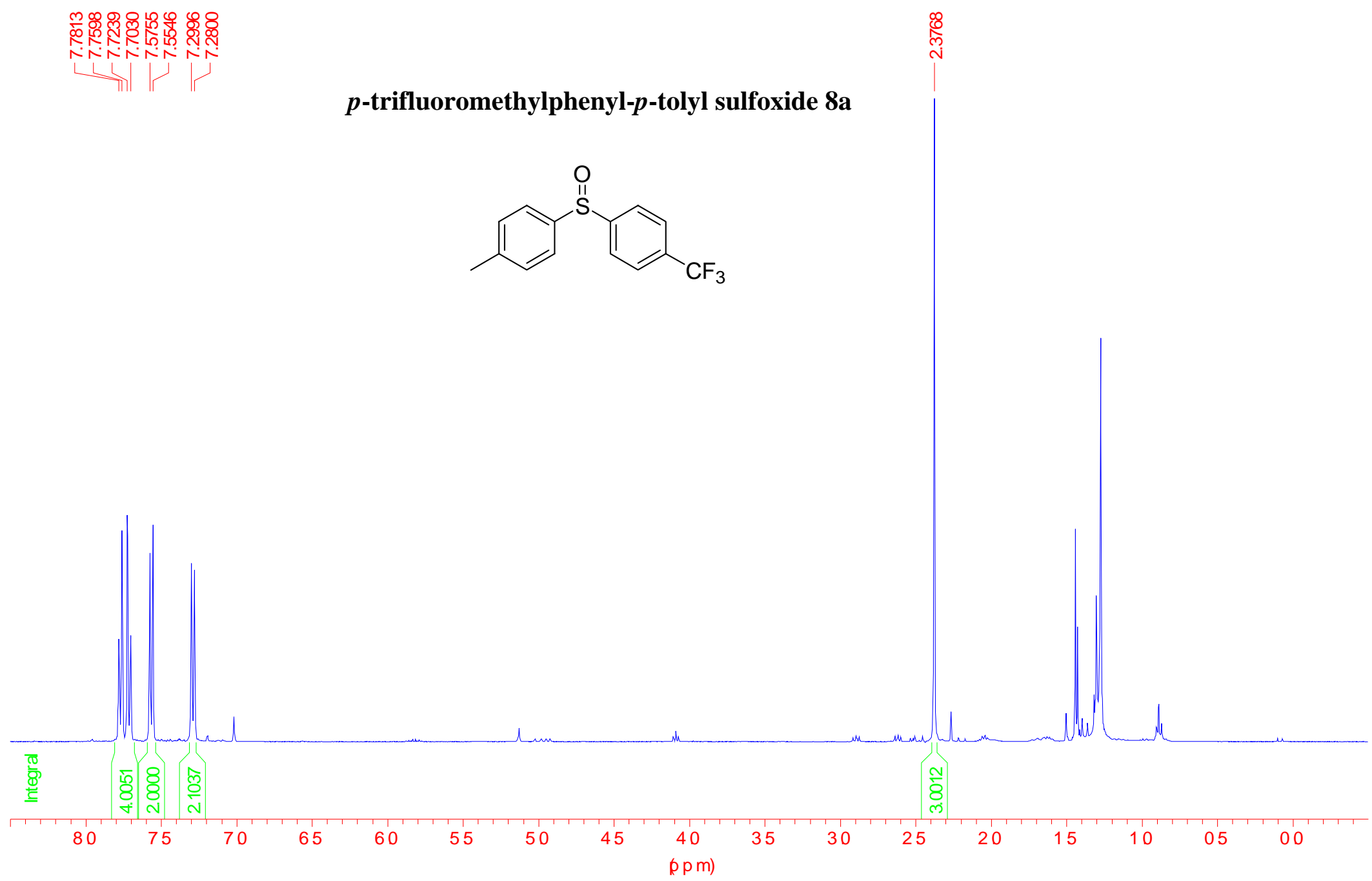




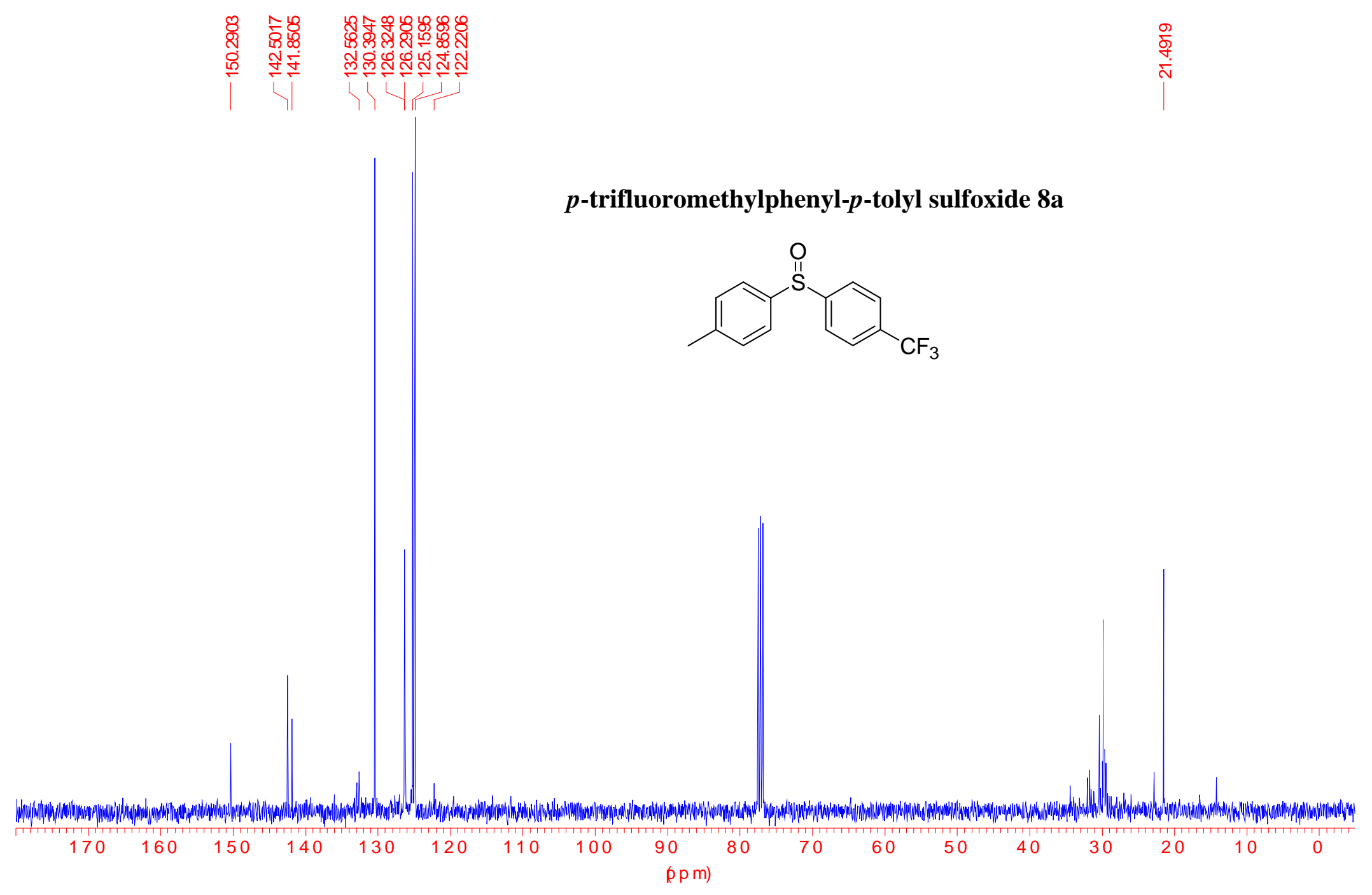



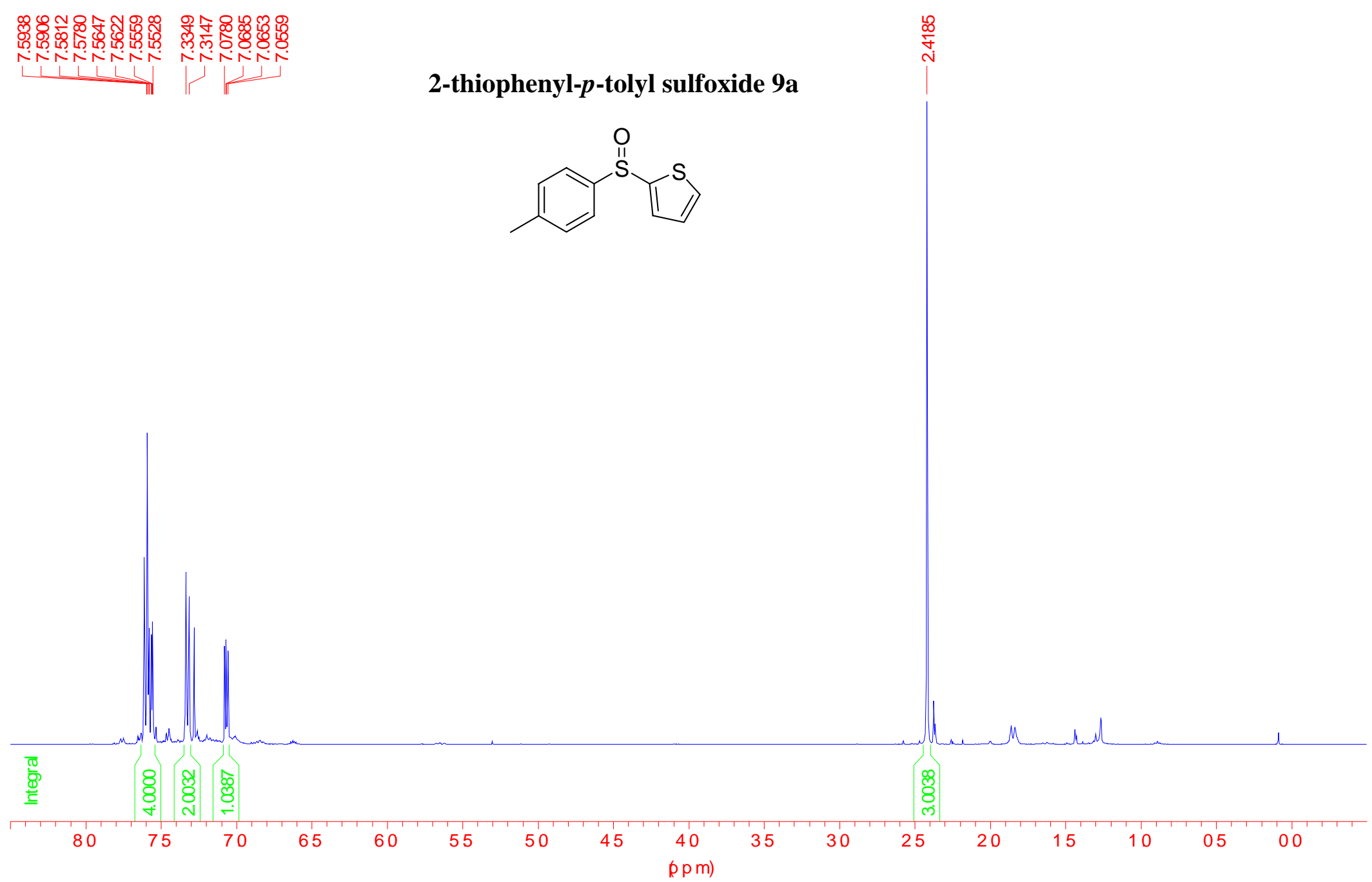


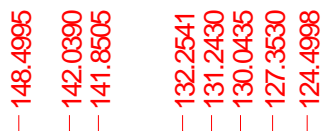

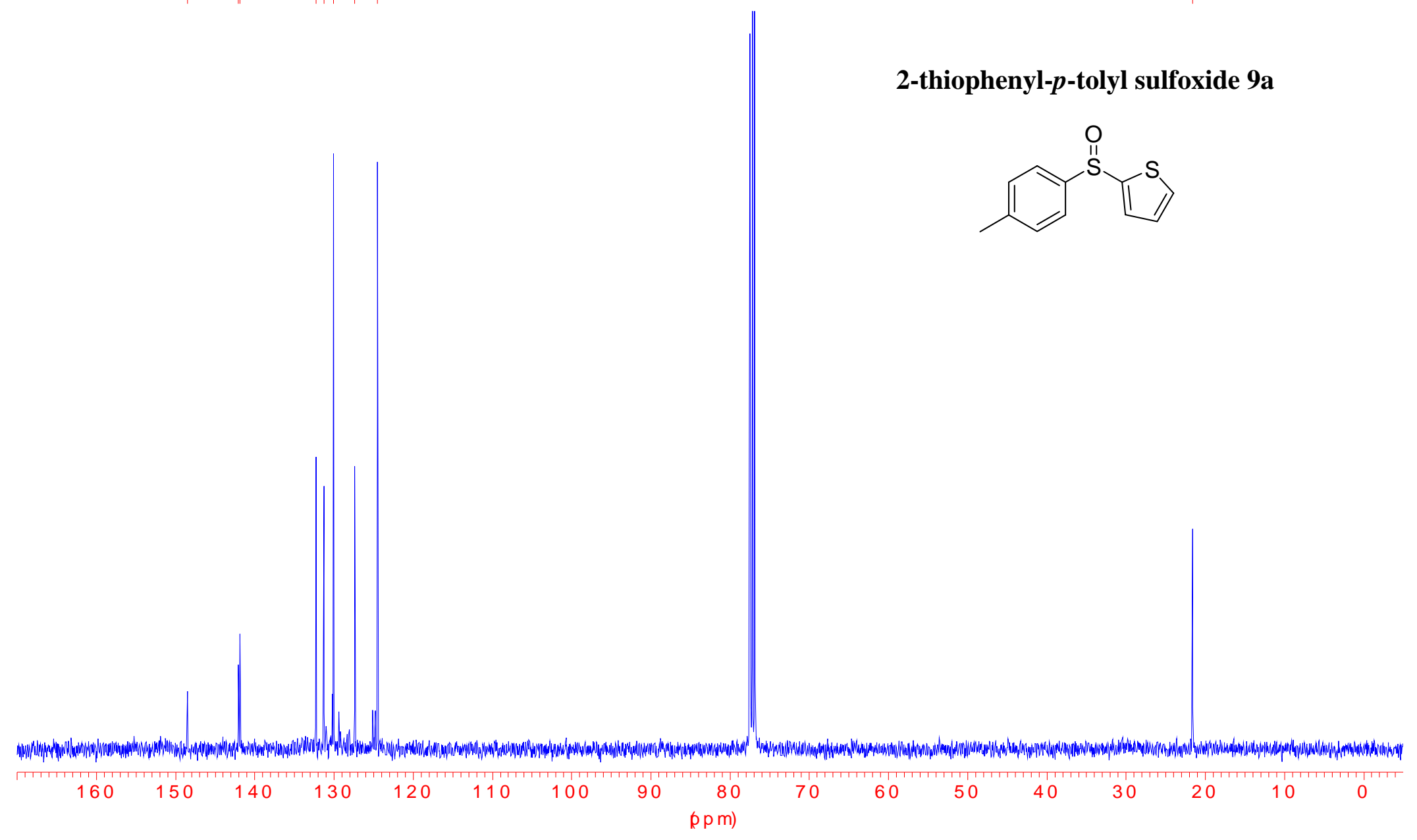



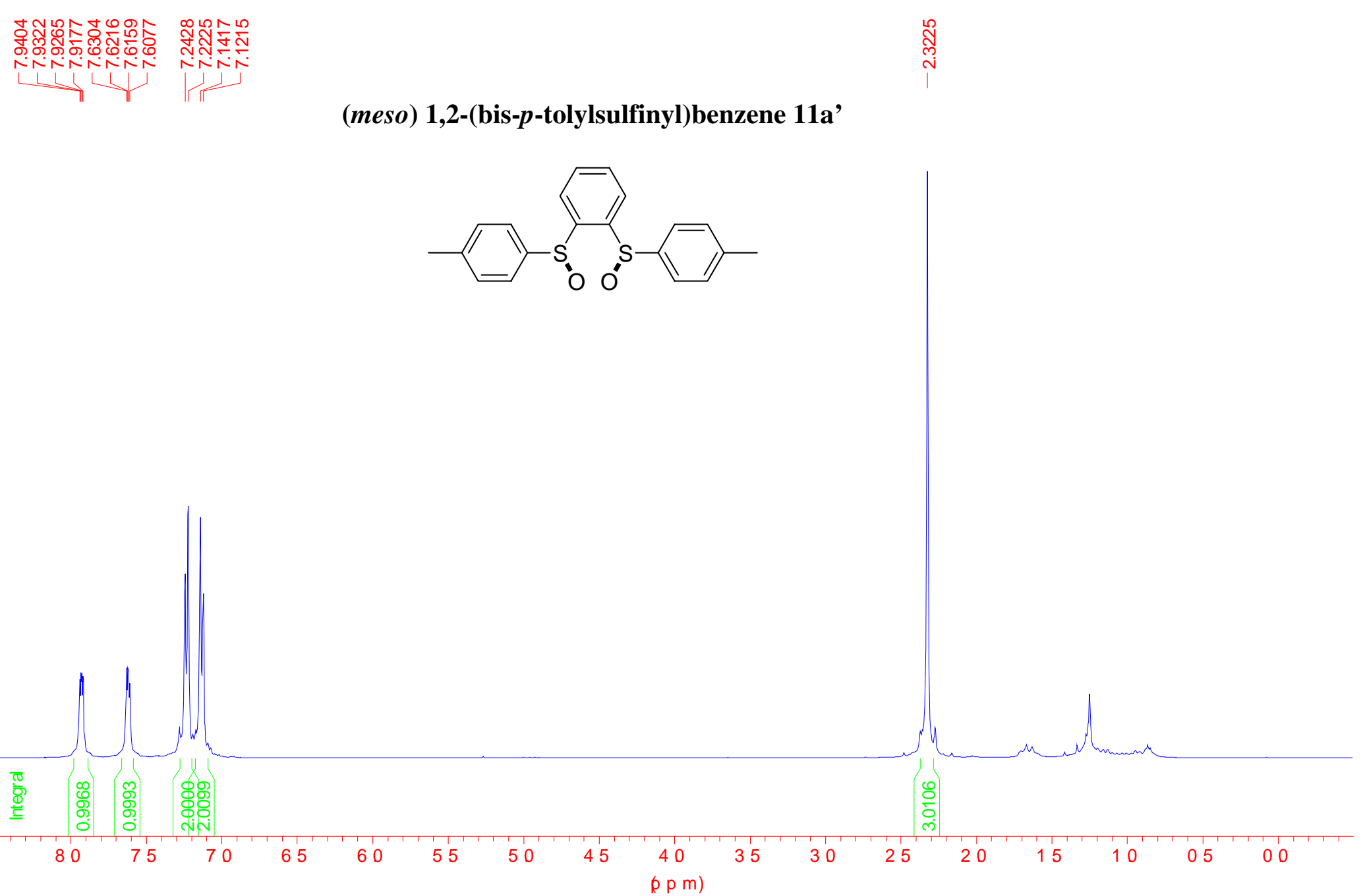


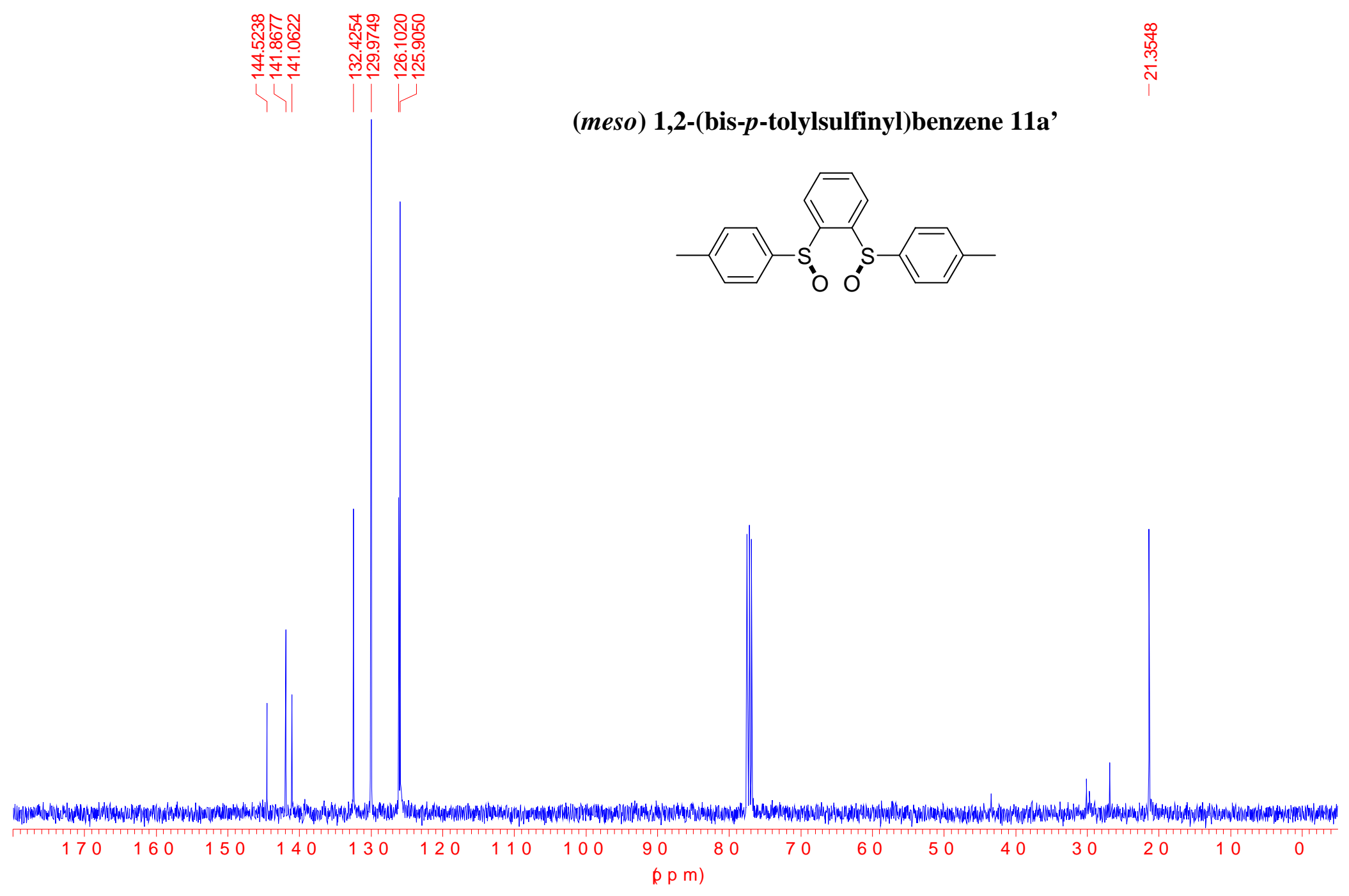




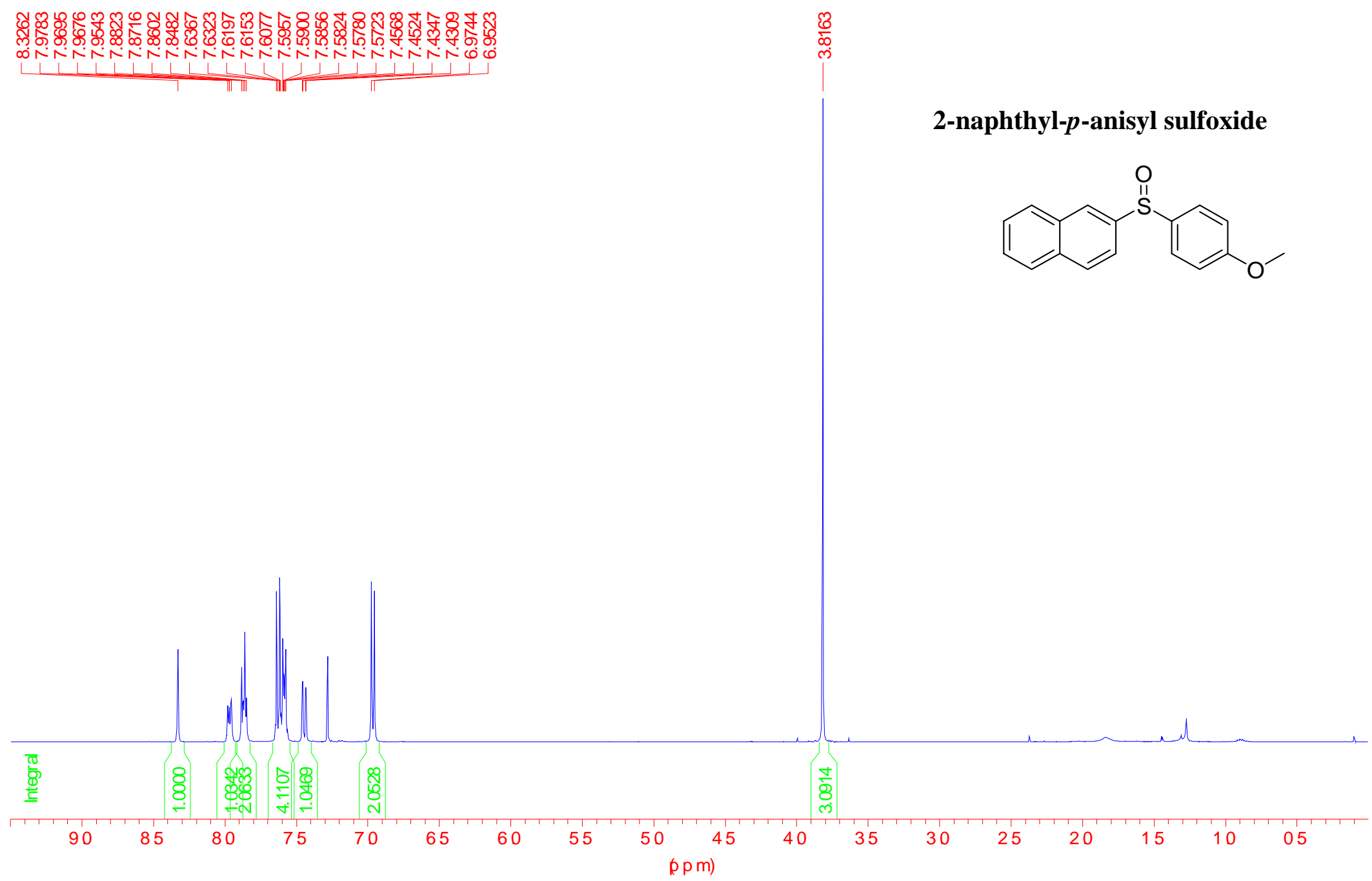




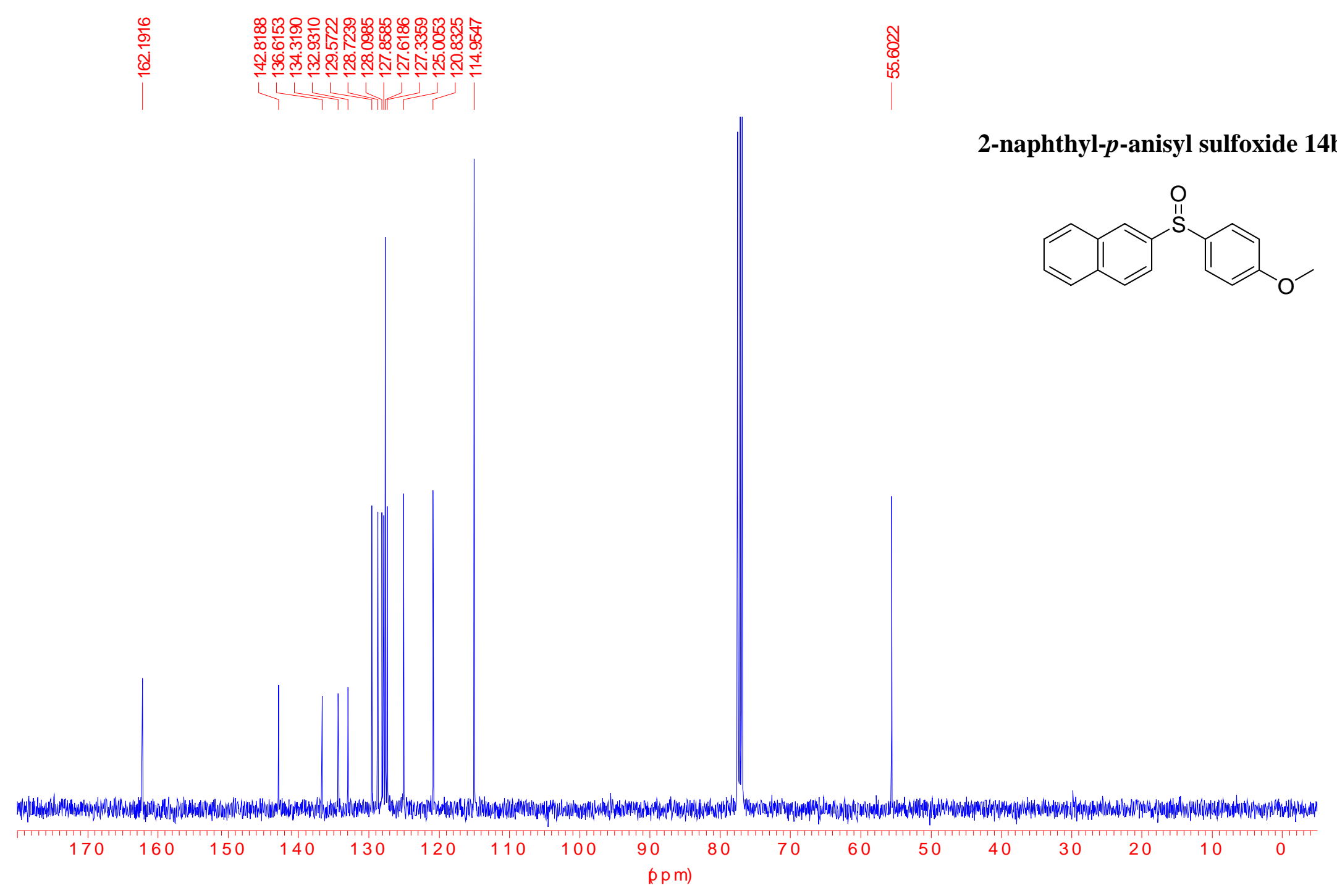




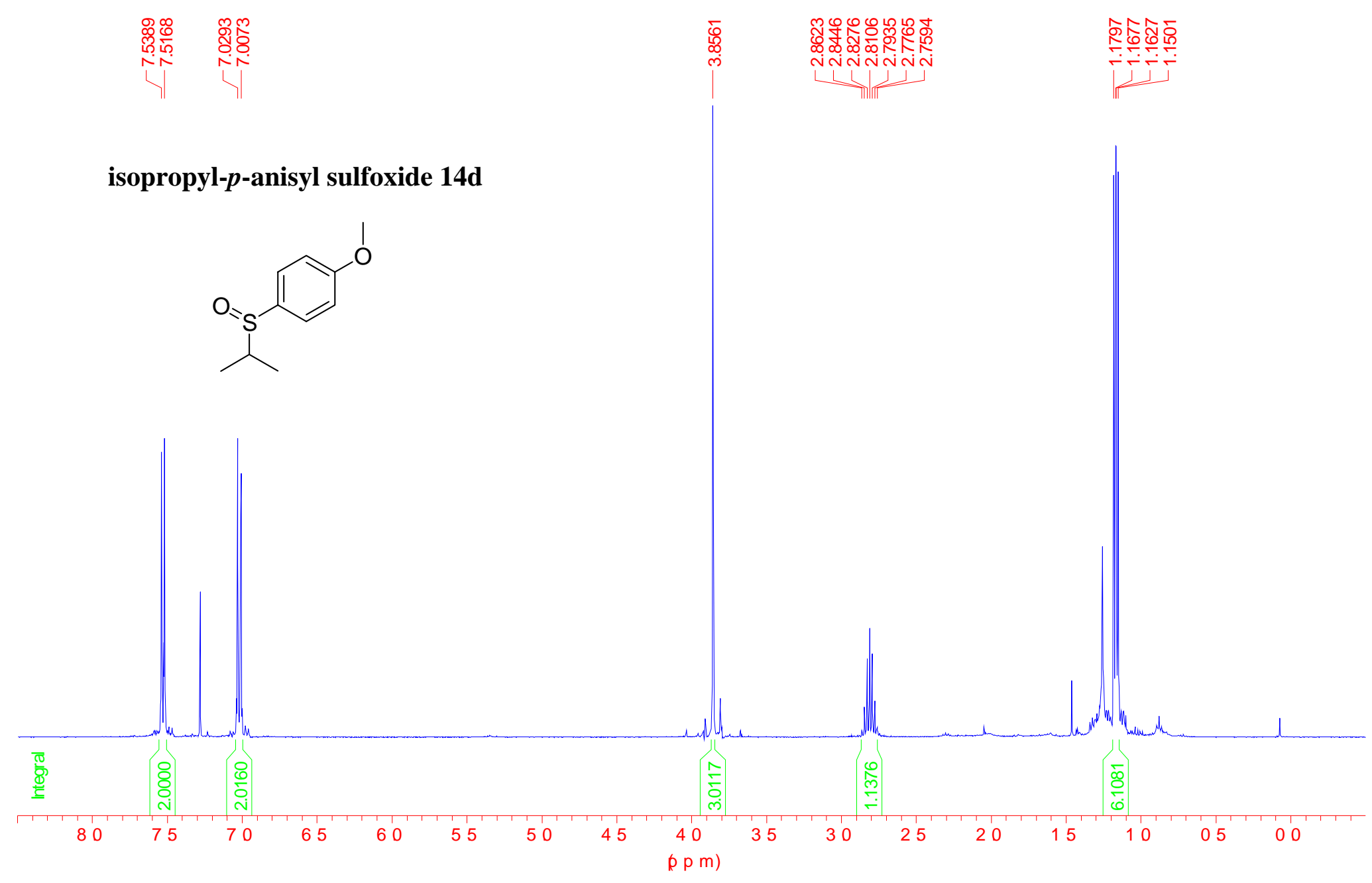




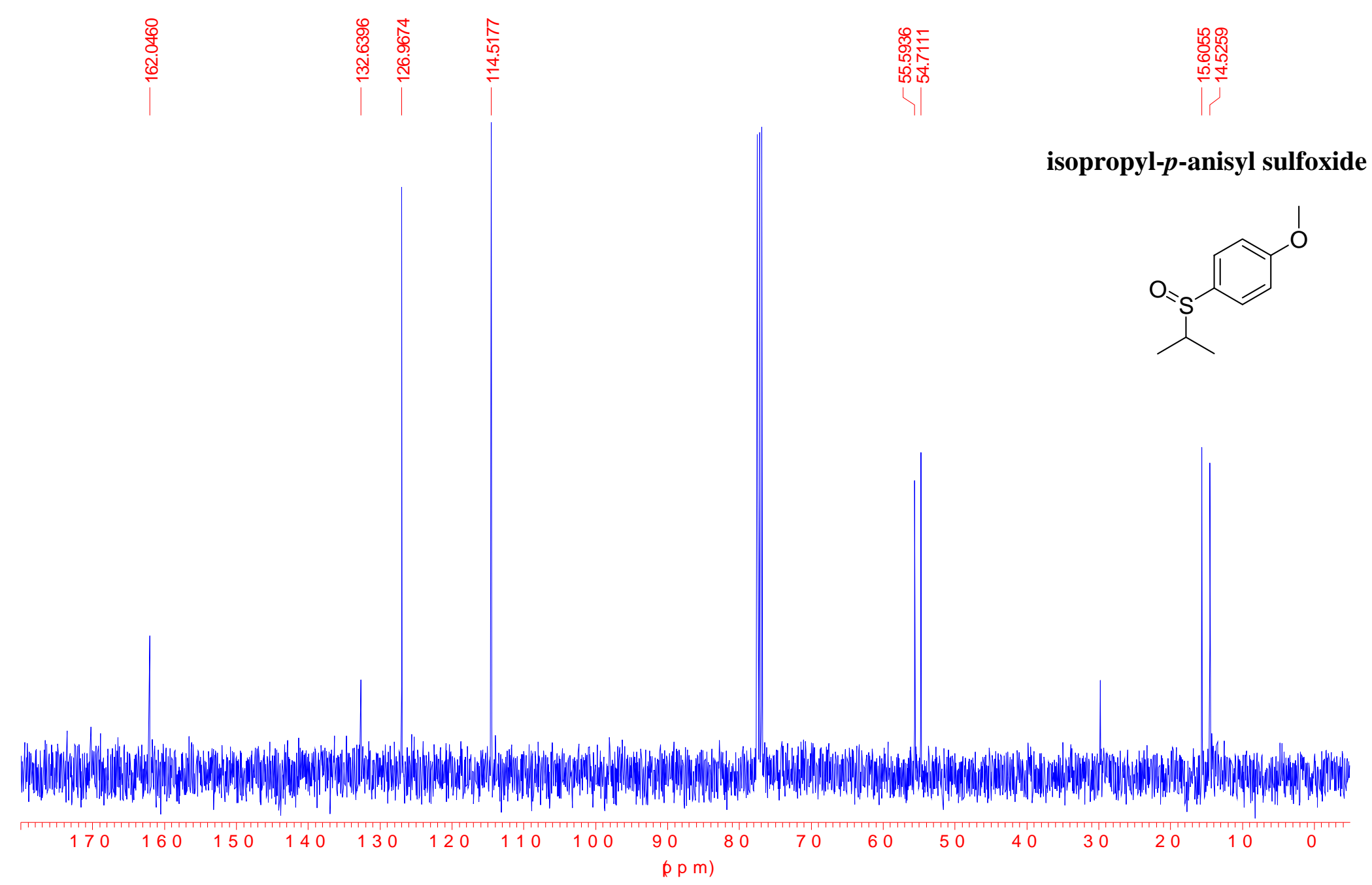




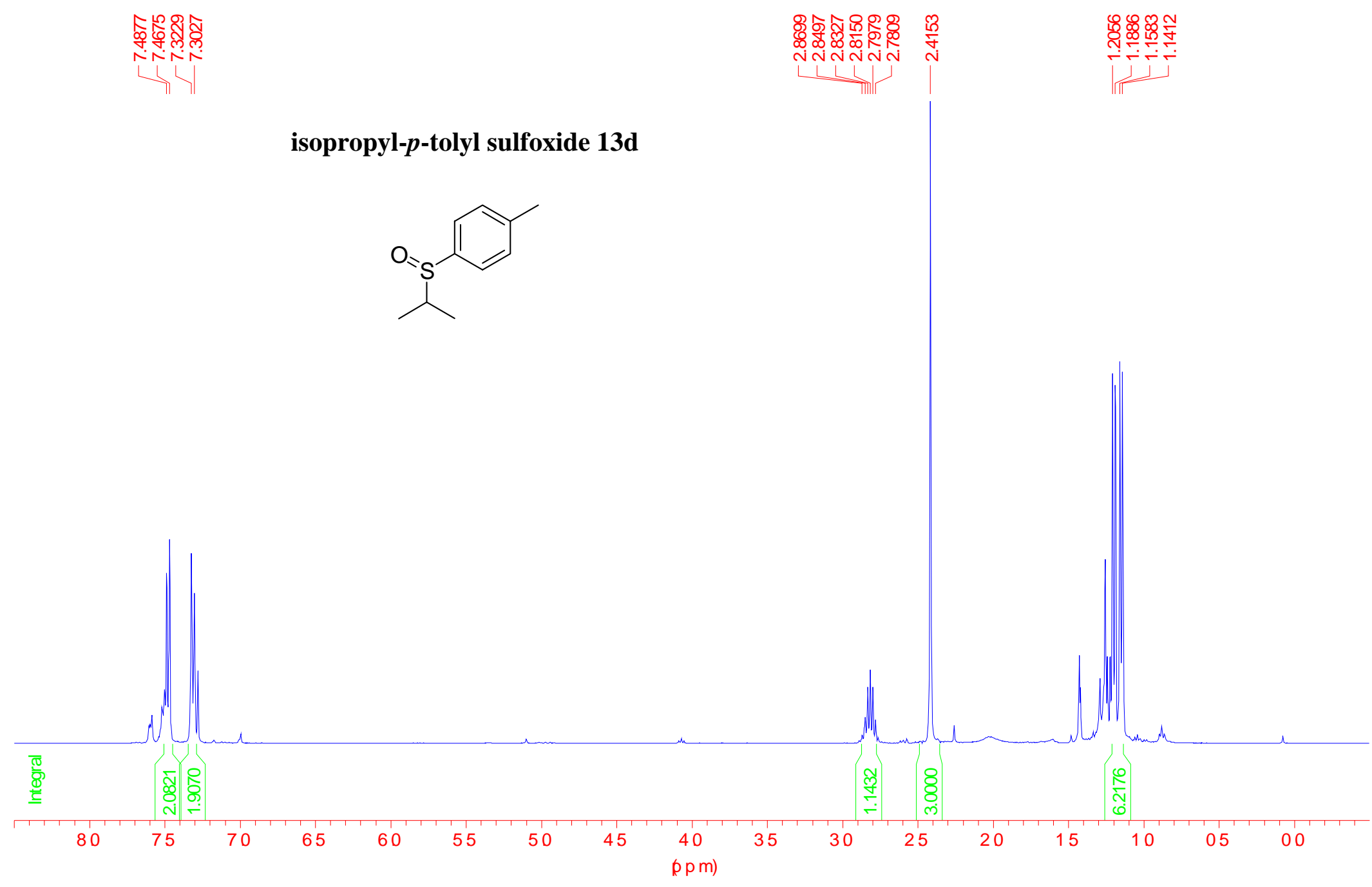




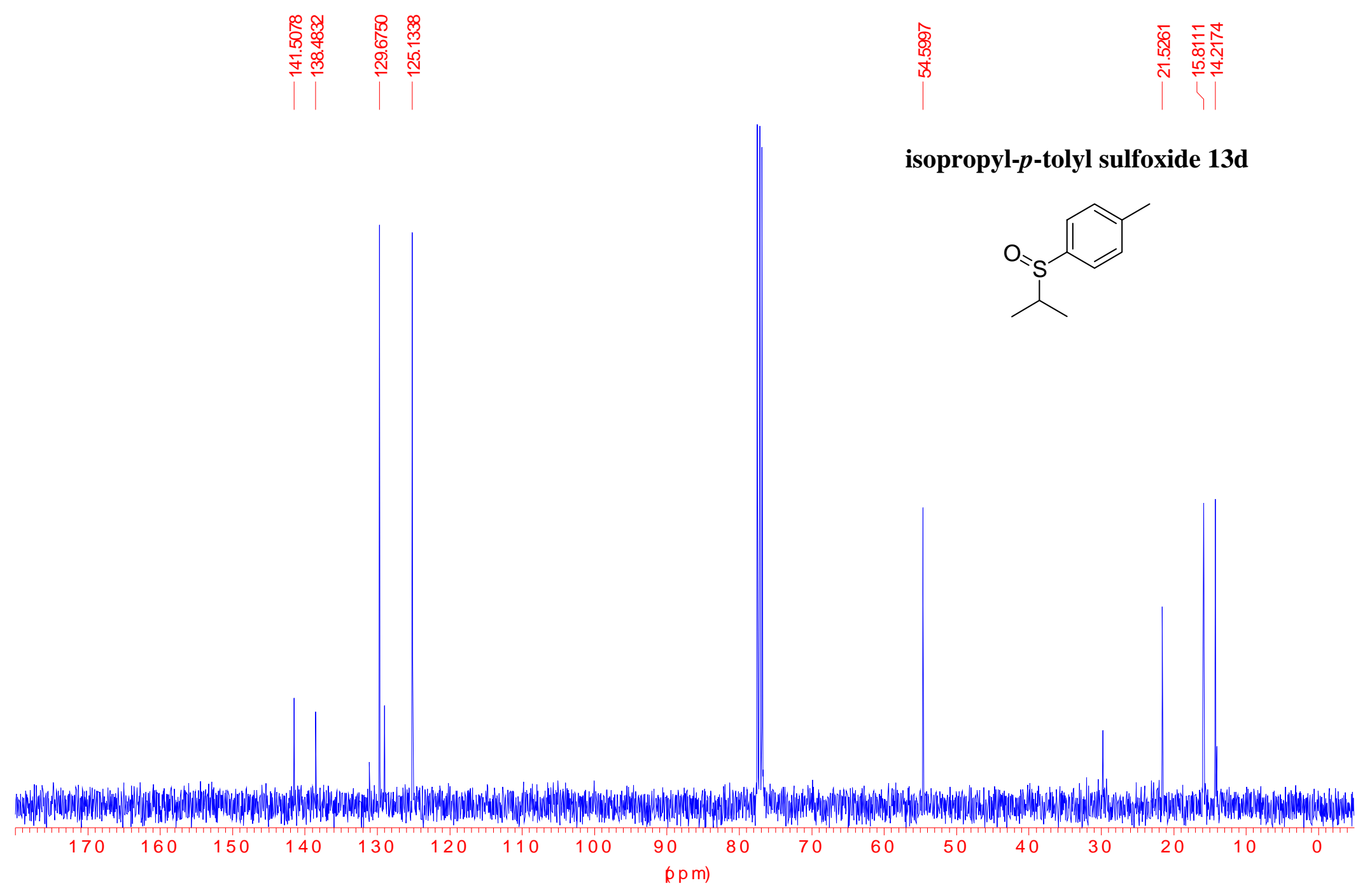


(E)-3-[4-(tolyl-4-sulfinyl)-phenyl]-acrylic acid tert-butyl ester 16
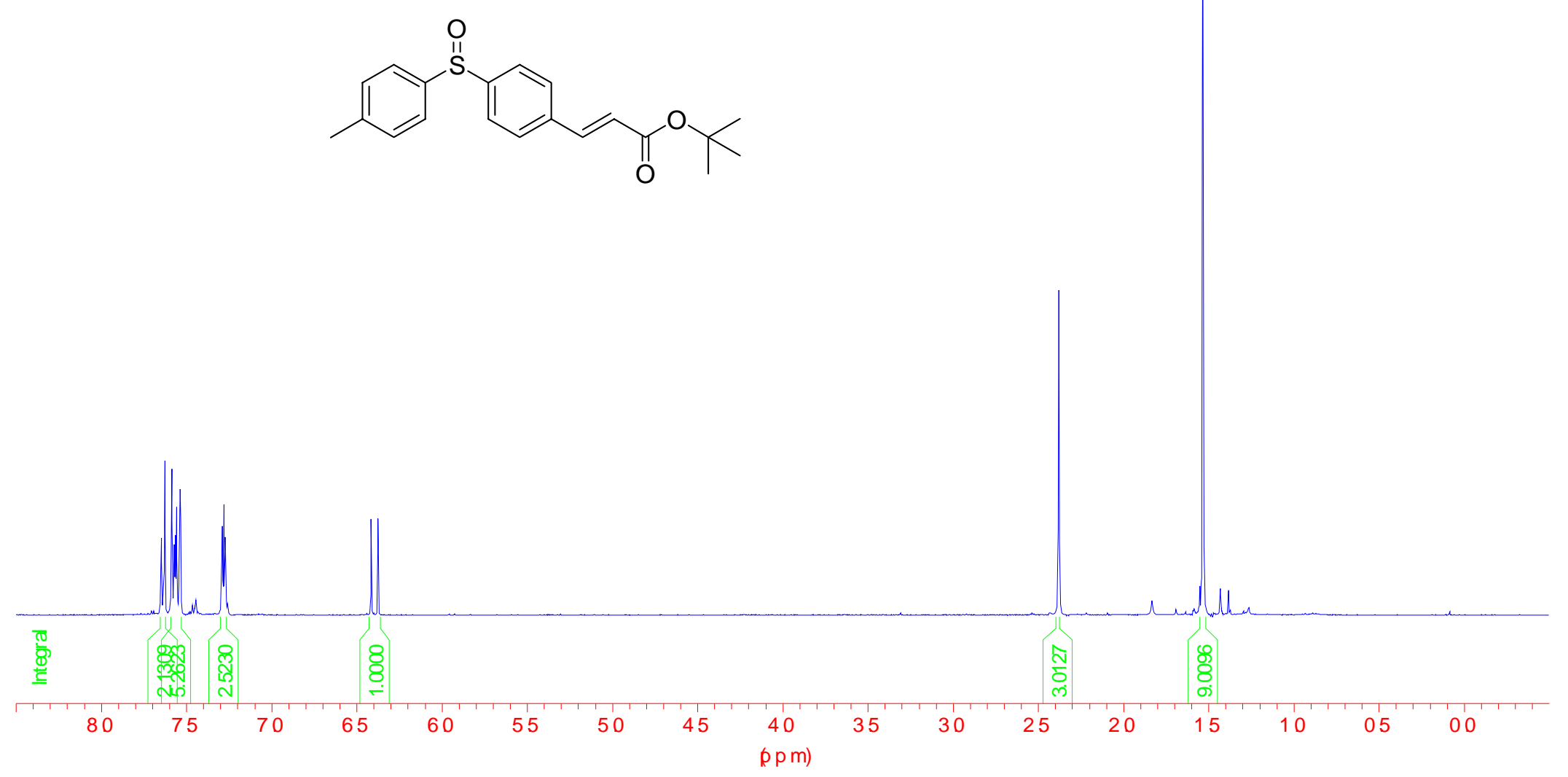


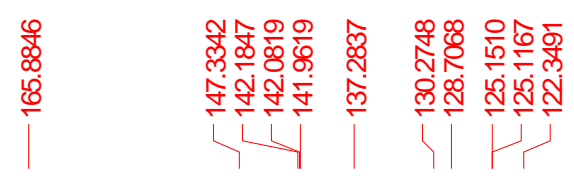

(E)-3-[4-(tolyl-4-sulfinyl)-phenyl]-acrylic acid tert-butyl ester 16

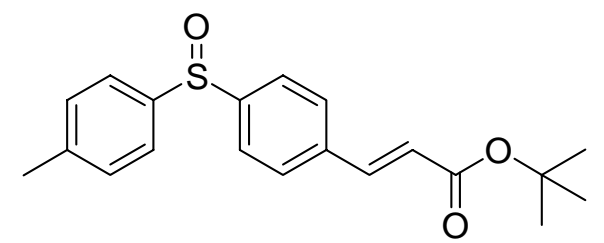

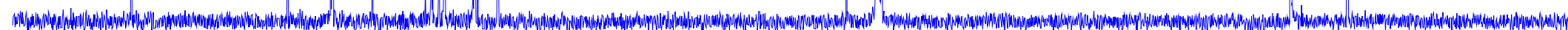

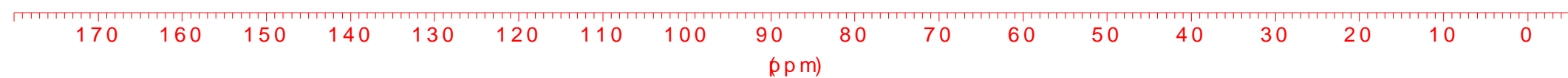

